\title{
Semiclassical probability of radiation of twisted photons in the ultrarelativistic limit
}

\author{
O. V. Bogdanov, ${ }^{1,2, *}$ P. O. Kazinski, ${ }^{1, \dagger}$ and G. Yu. Lazarenko ${ }^{1, *}$ \\ ${ }^{1}$ Faculty of Physics, Tomsk State University, Tomsk, 634050 Russia \\ ${ }^{2}$ Division for Mathematics and Computer Sciences, Tomsk Polytechnic University, Tomsk, 634050 Russia
}

(Received 26 March 2019; published 21 June 2019)

\begin{abstract}
The semiclassical general formula for the probability of radiation of twisted photons by ultrarelativistic scalar and Dirac particles moving in the electromagnetic field of a general form is derived. This formula is the analog of the Baier-Katkov formula for the probability of radiation of one plane wave photon with the quantum recoil taken into account. The derived formula is used to describe the radiation of twisted photons by charged particles in undulators and laser waves. Thus, the general theory of undulator radiation of twisted photons and radiation of twisted photons in the nonlinear Compton process is developed with account for the quantum recoil. The explicit formulas for the probability to record a twisted photon are obtained in these cases. In particular, we found that the quantum recoil and spin degrees of freedom increase the radiation probability of twisted photons in comparison with the formula for scalar particles without recoil. In the range of applicability of the semiclassical formula, the selection rules for undulator radiation established in the purely classical framework are not violated. The manifestation of the blossoming out rose effect in the nonlinear Compton process in a strong laser wave with circular polarization and in the wiggler radiation is revealed. Several examples are studied: the radiation of megaelectron-volt twisted photons by $180 \mathrm{GeV}$ electrons in the wiggler, the radiation of twisted photons by $256 \mathrm{MeV}$ electrons in strong electromagnetic waves produced by the $\mathrm{CO}_{2}$ and Ti:Sa lasers, and the radiation of $\mathrm{MeV}$ twisted photons by $51.1 \mathrm{MeV}$ electrons in the electromagnetic wave generated by the free-electron laser with photon energy $1 \mathrm{keV}$.
\end{abstract}

DOI: 10.1103/PhysRevD.99.116016

\section{INTRODUCTION}

Nowadays, the Baier-Katkov (BK) semiclassical method [1-4] is a standard tool to describe radiation of plane wave photons by ultrarelativistic charged particles in external electromagnetic fields of a general form. This method effectively includes the quantum recoil experienced by a charged particle in radiating one hard photon and is applicable for the energies of radiated photons right up to the energy of the radiating particle (for other semiclassical methods see, e.g., Refs. [5-10]). The BK method is realized in several computer codes [11-14] and proved to be very successful [4,15-22]. A comparison of the radiation probability obtained by this method with the exact QED results, when they are obtainable, reveals a spectacular agreement $[2-4,16]$. We use this method to derive the radiation probability of one twisted photon [23-32] by an ultrarelativistic charged particle with account for the quantum recoil. In the case of negligible quantum recoil, the obtained general formula reduces to the one derived in Ref. [33].

\footnotetext{
*bov@tpu.ru

tkpo@phys.tsu.ru

”laz@phys.tsu.ru
}

According to the BK method, in the ultrarelativistic limit, the one-photon radiation probability can be calculated by means of a formula resembling the classical formula for the intensity of radiation $[34,35]$. One does not need to solve the Dirac or Klein-Gordon equations in the given external fields but just has to find the solution of the Lorentz equations in these fields. The spin degrees of freedom are characterized by the spin vector, and its evolution is governed by the Bargmann-Michel-Telegdi equation. If the radiation probability is summed over spin polarizations of the escaping particle and averaged over spin polarizations of the incoming one, the dynamics of the spin vector are irrelevant for evaluation of the radiation probability. All that drastically simplifies the calculations of radiation probability. Of course, there are certain limitations of this semiclassical method. The complete list of them is presented in Sec. II. The main idea standing behind our derivation of the radiation probability of twisted photons is to find the approximate expression for the product of radiation amplitudes using the procedure developed in Refs. [1-4] and to integrate it over the azimuth angles of photon momenta with the corresponding weights. The last step creates the twisted photon in the out state. 
Having derived the general formula, we employ it to investigate the radiation probability of twisted photons by electrons in undulators (Sec. III) and strong laser pulses (Sec. IV). Currently, the twisted photons of different spectral ranges are used in fundamental science and technology [27-32]. In the optical range and below, the detectors were designed that allow one to decompose an arbitrary electromagnetic radiation into twisted photons [36-40]. Even in the x-ray spectral range, the twisted photons can be directly detected [41]. The formulas we obtain are aimed to describe correctly the radiation of hard twisted photons with mega-electron-volt energies and above that are not accessible for an immediate observation. The hard twisted photons can be employed to study the properties of nuclear matter by exciting higher multipole transitions in nuclei and hadrons (see, e.g., the discussions in Refs. $[24,25,42,43])$. Rather recently, it was shown that hard twisted photons can be generated in the inverse Compton scattering of low-energy twisted photons $[24,25]$, in channeling radiation $[44,45]$, and strong laser pulses [42,46-49]. In Sec. III, we show that mega-electronvolt photons can be produced by $180 \mathrm{GeV}$ electrons in helical wigglers. Besides, we develop a general theory of undulator radiation of twisted photons with the quantum recoil taken into account. In particular, we show that the selection rules for the forward radiation of twisted photons by helical undulators $[33,42,46,50-56]$ are not affected by the quantum recoil, at least, in the domain of applicability of the semiclassical method. Then, in Sec. IV, we revisit the problem of radiation of twisted photons by electrons in laser waves studied in Refs. [42,46,47]. We generalize the results of Refs. $[42,46,47]$ to the case of laser waves with an arbitrary amplitude envelope and include the influence of quantum recoil. Thus, we develop a general theory of twisted photon production in the nonlinear Compton process. Of course, it is just another description of the nonlinear Compton process usually formulated in terms of plane wave photons [2-4,15,17,19-21,57-68]. In Sec. IV, we apply this general theory and describe the radiation of twisted photons by electrons in strong laser pulses produced by the free-electron laser (FEL) and $\mathrm{CO}_{2}$ and Ti:Sa lasers. The obtained general formulas can be implemented in computer codes to describe the radiation of twisted photons by ultrarelativistic charged particles in electromagnetic fields of a rather general configuration, in particular, in channeling.

The paper is organized as follows. In Sec. II, we derive the general semiclassical formulas for the one-photon radiation probability of twisted photons by scalar and Dirac particles and discuss their applicability conditions. In Sec. III, we elaborate a general theory of undulator radiation of twisted photons, taking into account the quantum recoil undergone by a charged particle in radiating a photon. We also obtain a simple estimate for the number of radiated twisted photons and specialize the general applicability conditions to the case of undulator radiation. As expected, the wiggler radiation of lower harmonics in which the main part of twisted photons is radiated has a clear imprint of the "blossoming out rose" effect [9]. In Sec. IV, we consider the radiation of twisted photons by charged particles in an intense laser wave of a circular polarization. In particular, we trace the manifestation of the blossoming out rose effect in this radiation. Several examples are presented there. Some cumbersome calculations are removed to Appendixes A and C. The estimates of the transverse size of a particle wave packet are presented in Appendix B. In the Conclusion, we summarize the results.

We use the system of units such that $\hbar=c=1$ and $e^{2}=4 \pi \alpha$, where $\alpha \approx 1 / 137$ is the fine structure constant. Besides, we vastly use the notation introduced in Ref. [33].

\section{GENERAL FORMULAS}

Let us begin with the case of a stationary external magnetic field. The generalization to the case of a general electromagnetic field will be given below. In the presence of the electromagnetic field, the following process is possible,

$$
e_{i}^{-} \rightarrow \gamma_{\alpha}+X,
$$

where $e_{i}^{-}$is the initial electron in the state $i, \gamma_{\alpha}$ is the twisted photon [23-27,69-73] recorded by the detector in the state $\alpha$, and $X$ denotes the rest of particles that are not recorded by the detector. Notice that such a detector can be not only a specially designed detector of twisted photons [36-41] but a molecule, an atom, a nucleus, etc. The probability of such an inclusive process equals

$$
w(\alpha ; i)=\sum_{X}\left\langle i\left|\hat{U}_{-\infty, \infty}\right| X ; \gamma_{\alpha}\right\rangle\left\langle\gamma_{\alpha} ; X\left|\hat{U}_{\infty,-\infty}\right| i\right\rangle,
$$

where $\hat{U}$ is the evolution operator of QED in the presence of the external field (see, e.g., Refs. [59,74]). In the first Born approximation with the exact account for the external electromagnetic field, the final state contains only one electron,

$$
w(\alpha ; i) \approx \sum_{f}\left\langle i\left|\hat{U}_{-\infty, \infty}\right| f ; \gamma_{\alpha}\right\rangle\left\langle\gamma_{\alpha} ; f\left|\hat{U}_{\infty,-\infty}\right| i\right\rangle,
$$

where $f$ characterizes the final electron state. In this approximation, using the completeness relation, we obtain

$$
w(\alpha ; i)=\left\langle i\left|\hat{U}_{-\infty, \infty}\right| \gamma_{\alpha}\right\rangle\left\langle\gamma_{\alpha}\left|\hat{U}_{\infty,-\infty}\right| i\right\rangle
$$

i.e., the probability of process (1) is equal to the average number of photons in the final state $\alpha$. According to (A5), the twisted photon state can be decomposed into the plane wave ones [23-27,69-73]. Then, 
$w(\alpha ; i)=\sum_{\mathbf{k}_{1}, \mathbf{k}_{2}} \Lambda_{\alpha ; s, \mathbf{k}_{2}}^{*} \Lambda_{\alpha ; s, \mathbf{k}_{1}}\left\langle i\left|\hat{U}_{-\infty, \infty}\right| s, \mathbf{k}_{2}\right\rangle\left\langle s, \mathbf{k}_{1}\left|\hat{U}_{\infty,-\infty}\right| i\right\rangle$ where $\Lambda_{\alpha ; s, \mathbf{k}}$ are the coefficients of expansion (A5). Therefore, in the first Born approximation, $w(\alpha ; i)$ can be found from the matrix element for the plane wave photons (see the notation in Ref. [4])

$$
C\left(\mathbf{k}_{2}, \mathbf{k}_{1}\right):=\frac{e^{2}}{(2 \pi)^{3} 2 V k_{0}}\left\langle i\left|\int_{-\infty}^{\infty} d t_{1} d t_{2} e^{i k_{0}\left(t_{1}-t_{2}\right)} \hat{M}^{\dagger}\left(t_{2}, \mathbf{k}_{2}\right) \hat{M}\left(t_{1}, \mathbf{k}_{1}\right)\right| i\right\rangle
$$

which should be integrated over the azimuth angles of the vectors $\mathbf{k}_{1,2}$ with the corresponding phase factors as in (A5). Notice that we work in the coordinate system adapted to the detector of twisted photons with the axis directed along the unit vector $\mathbf{e}_{3}$ (see, for details, Ref. [33]). The unit vectors $\left\{\mathbf{e}_{1}, \mathbf{e}_{2}, \mathbf{e}_{3}\right\}$ of this coordinate system constitute a right-hand triple. The perpendicular components of a vector are those lying in the plane spanned by $\left\{\mathbf{e}_{1}, \mathbf{e}_{2}\right\}$. The photons are supposed to lie on the mass shell

$$
k_{0}=\sqrt{k_{1 \perp}^{2}+k_{13}^{2}}=\sqrt{k_{2 \perp}^{2}+k_{23}^{2}}
$$

and

$$
k_{1 \perp}=k_{2 \perp}, \quad k_{13}=k_{23} ;
$$

i.e., the photon momenta $\mathbf{k}_{1,2}$ differ only by the azimuth angles. The helicities of photons with momenta $\mathbf{k}_{1,2}$ are the same.

We will evaluate (6) using the approximation introduced in Refs. [1-4]. Recall that it is assumed in this approximation that:

(1) The charged particle is ultrarelativistic, i.e., the Lorentz factor $\gamma \gg 1$ and $\varkappa / \gamma \ll 1$, where $\varkappa=\max (1, K), K=\left\langle\beta_{\perp}\right\rangle \gamma$ is the undulator strength parameter, and $\left\langle\beta_{\perp}\right\rangle$ is a typical value of the velocity component perpendicular to the detector axis.

(2) The particle wave packet is sufficiently narrow in the momentum space.

(3) The size of the wave packet in the configuration space is small in comparison with the typical scale of variation of the external electromagnetic field in space.

(4) $\mathbf{n}_{1,2}:=\mathbf{k}_{1,2} / k_{0}$ lie inside of the cone directed along the detector axis (the axis 3 ) with the opening of order $2 \varkappa / \gamma$; i.e., we consider the region where the main part of radiation is concentrated.

Below, the additional restriction related to the fact that we consider the radiation of twisted photons will appear. In contrast to the case of radiation of plane wave photons, we have to know $C\left(\mathbf{k}_{2}, \mathbf{k}_{1}\right)$ out of the diagonal, and so the additional assumptions will be needed.

If the above conditions are satisfied, then, in evaluating average (6) in the leading order in $1 / \gamma$, we can use the analog of the Thomas-Fermi approximation. Namely, the commutator

$$
\left|\left\langle\left[\hat{P}_{\mu}, \hat{A}_{\nu}\right]\right\rangle\right| /\left\langle\hat{\mathbf{P}}^{2}\right\rangle \sim \frac{H}{H_{0} \gamma^{2}} \ll 1, \quad \gamma \gg 1,
$$

is small for large particle energies. Here, $H$ is a typical magnitude of the electromagnetic field, $H_{0}$ is the critical (Schwinger) field, and $\hat{P}_{\mu}=\hat{p}_{\mu}-e A_{\mu}(\hat{\mathbf{x}})$. Hence, the noncommutativity of operators $\hat{\mathbf{x}}$ and $\hat{\mathbf{P}}$ entering into the operators $\hat{M}^{\dagger}\left(t_{2}, \mathbf{k}_{2}\right), \hat{M}\left(t_{1}, \mathbf{k}_{1}\right)$ can be neglected. At the same time, the noncommutativity of $\hat{\mathbf{P}}$ and $e^{-i \mathbf{k} \hat{\mathbf{x}}}$ in $\hat{M}$ cannot be ignored because the exponent is a rapidly varying function for $|\mathbf{k}| \sim \varepsilon$, where $\varepsilon$ is the energy of a charged particle.

\section{A. Scalar particle}

For a scalar charged particle, we have [4]

$$
\begin{aligned}
\hat{M}\left(t_{1}, \mathbf{k}_{1}\right) & =\hat{P}_{0}^{-1 / 2}\left(\mathbf{f}_{1}^{*} \hat{\mathbf{P}}\left(t_{1}\right)\right) e^{-i \mathbf{k}_{1} \hat{\mathbf{x}}\left(t_{1}\right)} \hat{P}_{0}^{-1 / 2}, \\
\hat{M}^{\dagger}\left(t_{2}, \mathbf{k}_{2}\right) & =\hat{P}_{0}^{-1 / 2}\left(\mathbf{f}_{2} \hat{\mathbf{P}}\left(t_{2}\right)\right) e^{i \mathbf{k}_{2} \hat{\mathbf{x}}\left(t_{2}\right)} \hat{P}_{0}^{-1 / 2},
\end{aligned}
$$

where $\mathbf{f}_{1,2}=\mathbf{f}\left(\mathbf{k}_{1,2}\right)$ are the polarization vectors of physical photons. All the operators in (10) are given in the Heisenberg representation with the Hamiltonian

$$
\hat{H}=\sqrt{\hat{\mathbf{P}}^{2}+m_{e}^{2}}+e A_{0}(\hat{\mathbf{x}}) .
$$

Henceforth, we perform our calculations in the Coulomb gauge. Notice that $A_{0}=0$ in the case of time-independent magnetic field. However, we leave $A_{0}$ intact since its presence influences the derivation only in one point [Eq. (22)], which we shall discuss separately below. To avoid additional complications with the vacuum definition (see, e.g., Refs. [59,74-76]), we suppose that

$$
\left|e A_{0}\right| \lesssim m_{e}
$$

which is fulfilled for all the electromagnetic fields achievable at the present moment in laboratories. Therefore, $P_{0}=$ $m_{e} \gamma \approx \varepsilon$ up to the terms of order $1 / \gamma$. 
Let us carry the exponent entering into $\hat{M}^{\dagger}\left(t_{2}, \mathbf{k}_{2}\right)$ to the right and the one entering into $\hat{M}\left(t_{1}, \mathbf{k}_{1}\right)$ to the left,

$$
\begin{aligned}
& \hat{M}^{\dagger}\left(t_{2}, \mathbf{k}_{2}\right) \hat{M}\left(t_{1}, \mathbf{k}_{1}\right) \\
& \quad=\hat{P}_{02}^{-1 / 2}\left(\mathbf{f}_{2} \hat{\mathbf{P}}_{2}\right) \hat{P}_{02}^{\prime-1 / 2} e^{i \mathbf{k}_{2} \hat{\mathbf{x}}_{2}} e^{-i \mathbf{k}_{1} \hat{\mathbf{x}}_{1}} \hat{P}_{01}^{\prime-1 / 2}\left(\mathbf{f}_{1}^{*} \hat{\mathbf{P}}_{1}\right) \hat{P}_{01}^{-1 / 2},
\end{aligned}
$$

where

$$
\hat{P}_{01,2}^{\prime}=P_{0}\left(\hat{\mathbf{P}}_{1,2}^{\prime}\right), \hat{\mathbf{P}}_{1,2}^{\prime}:=\hat{\mathbf{P}}_{1,2}-\mathbf{k}_{1,2},
$$

and $\hat{\mathbf{P}}_{1,2} \equiv \hat{\mathbf{P}}\left(t_{1,2}\right)$. Then, we transform the operator expression

$$
\begin{aligned}
e^{i \mathbf{k}_{2} \hat{\mathbf{x}}_{2}} e^{-i \mathbf{k}_{1} \hat{\mathbf{x}}_{1}} & =e^{i \mathbf{k}_{\perp 2} \hat{\mathbf{x}}_{\perp 2}} e^{i k_{3} \hat{x}_{32}} e^{-i k_{3} \hat{x}_{31}} e^{-i \mathbf{k}_{\perp 1} \hat{\mathbf{x}}_{\perp 1}} \\
& =e^{i \mathbf{k}_{\perp 2} \hat{\mathbf{x}}_{\perp 2}} e^{i \hat{H} \tau}\left(e^{-i \hat{H} \tau}\right)_{\hat{P}_{3} \rightarrow \hat{P}_{3}-k_{3}} e^{-i \mathbf{k}_{\perp 1} \hat{\mathbf{x}}_{\perp 1}},
\end{aligned}
$$

where $\tau:=t_{2}-t_{1}$. To proceed, we assume that

$$
e^{-i \mathbf{k}_{\perp 1,2} \hat{\mathbf{x}}_{\perp 1,2}}|i\rangle \approx e^{-i \mathbf{k}_{\perp 1,2} \mathbf{x}_{\perp 1,2}}|i\rangle,
$$

where $\mathbf{x}_{1,2}$ is the average value of the corresponding operator with respect to the state $|i\rangle$. The approximate equality takes place, if

$$
k_{\perp} \sigma_{\perp} \ll 1
$$

where $\sigma_{\perp}$ is a typical transverse (with respect to the detector axis) size of the wave packet in the configuration space. The condition (17) is absent in considering the radiation of plane wave photons by the BK method. The reason is that the dependence of radiation probability for twisted photons on the transverse structure of a particle wave packet is stronger than for plane wave photons. It is not surprising as, in contrast to plane wave photons, the probability to record a twisted photon depends on the photon momentum component perpendicular to the detector axis.

Notice that condition (17) also arises in considering the radiation of twisted photons by a bunch of charged particles $[77,78]$. When condition (17) is satisfied, the probability of radiation of a twisted photon by the bunch of $N$ particles is the same as for one charged particle, multiplied by $N$ (incoherent radiation) or by $N^{2}$ (coherent radiation). To put it differently, the form of the wave packet does not affect the radiation spectrum of twisted photons in this case. It is clear that if (17) is violated the approximation we use cannot be employed. In that case, the probability of radiation of twisted photons depends severely on the form of the wave packet and is not determined only by the average values of the particle momentum and coordinate. In particular, none of the fine effects stemming from the form of the wave packet of a charged particle (see, e.g., Refs. [26,79-84]) can be reproduced by the semiclassical approach considered here. However, condition (17) cannot be strongly violated. It was shown in Refs. [77,78] that, in a general position, the coherent radiation produced by a smooth wave packet of a charged particle is strongly suppressed for $k_{\perp} \sigma_{\perp} \gtrsim 3$ due to destructive interference of the radiation amplitudes coming from different points of the wave packet.

Furthermore, we have

$$
\mid\left\langle\left[ e^{\left.\left.-i \mathbf{k}_{\perp 1,2} \hat{\mathbf{x}}_{\perp 1,2}, \hat{\mathbf{P}}\right]\right\rangle\left.|/|\left\langle\hat{\mathbf{P}}^{2}\right\rangle\right|^{1 / 2} \sim k_{0} \varkappa /(\varepsilon \gamma) \lesssim \varkappa / \gamma ;}\right.\right.
$$

i.e., up to the terms of order $x / \gamma$, we can carry these exponents through the operators entering into $\hat{M}_{2}^{\dagger}$ and $\hat{M}_{1}$ and make use of (16). Now, take into account that

$$
\begin{aligned}
& \sqrt{\left(P_{3}-k_{3}\right)^{2}+P_{\perp}^{2}+m^{2}} \\
& \approx\left(P_{0}-k_{0}\right)\left(1+\frac{k_{0} P_{0}-k_{3} P_{3}}{\left(P_{0}-k_{0}\right)^{2}}-\frac{k_{\perp}^{2}}{2\left(P_{0}-k_{0}\right)^{2}}\right) .
\end{aligned}
$$

The approximation (19) is valid, provided

$$
\frac{P_{0} k_{0}(1-\mathbf{n} \dot{\mathbf{x}})}{\left(P_{0}-k_{0}\right)^{2}} \sim \frac{k_{0} / P_{0}}{\left(1-k_{0} / P_{0}\right)^{2}} \frac{\varkappa^{2}}{\gamma^{2}} \ll 1 .
$$

If $\varkappa / \gamma$ is small, estimate (20) holds up to $k_{0} \lesssim P_{0}$. Then, repeating the calculations presented in Ref. [4], we can write

$$
\begin{aligned}
& e^{-i k_{0} \tau} e^{i \hat{H} \tau}\left(e^{-i \hat{H} \tau}\right)_{\hat{P}_{3} \rightarrow \hat{P}_{3}-k_{3}} \\
& \quad=\operatorname{Texp}\left\{i \int_{0}^{\tau} d t\left(H-k_{0}-H_{\hat{P}_{3} \rightarrow \hat{P}_{3}-k_{3}}\right)\right\} .
\end{aligned}
$$

Therefore,

$e^{-i k_{0} \tau} e^{i \hat{H} \tau}\left(e^{-i \hat{H} \tau}\right)_{\hat{P}_{3} \rightarrow \hat{P}_{3}-k_{3}} \approx e^{-i \frac{\hat{P}_{0}}{\hat{P}_{0}-k_{0}}\left[k_{0} \tau-k_{3}\left(x_{32}-x_{31}\right)-k_{\perp}^{2} \tau /\left(2 \hat{P}_{0}\right)\right]}$,

where we have used the fact that, in the time-independent magnetic fields, $P_{0}=m \gamma$ is an integral of motion of the Lorentz equations. It is argued in Ref. [4] that approximation (22) is valid in the leading order in $\varkappa / \gamma$ for general nonstationary external electromagnetic fields as well, provided

$$
2 \pi /(T \varepsilon) \ll 1,
$$

where $T$ is a typical timescale or length scale of changing of the external electromagnetic fields. In that case, $\hat{P}_{0}$ should be replaced by $\hat{P}_{0 i}$ in (22), i.e., by the particle energy in the initial state $|i\rangle$ where the external fields are absent. This prescription ensures, in particular, that the right-hand side of (22) is invariant under translations in the spacetime. 
Disentangling expression (15) and taking into account the conditions 1-4 and Eqs. (9) and (17), we can replace the operators in matrix element (6) by their average values. In particular,

$$
\hat{P}_{01,2}^{\prime} \rightarrow P_{01,2}^{\prime}=P_{0}\left(\mathbf{P}_{1,2}-\mathbf{k}_{1,2}\right) \approx P_{01,2}-k_{0} .
$$

As for exponent (22), this replacement is justified when the dispersion of

$q k_{0} \int_{0}^{\tau_{r}} d t\left(1-n_{3} \beta_{3}\right) \approx q k_{0} \int_{0}^{\tau_{r}} d t \frac{1+n_{\perp}^{2} \gamma^{2}+\beta_{\perp}^{2} \gamma^{2}}{2 \gamma^{2}}$

is much less than unity, where $\tau_{r}$ is the radiation formation time in the laboratory frame, $n_{\perp}:=k_{\perp} / k_{0}$, and we neglected the last term in the exponent in (22) as it is small in comparison with the first two [see (44) below]. By the order of magnitude,

$$
\tau_{r} q k_{0} \frac{1+n_{\perp}^{2} \gamma^{2}+\beta_{\perp}^{2} \gamma^{2}}{2 \gamma^{2}} \sim N
$$

where $N$ is the number of periods of particle motion. Then, for an isoenergetic wave packet, we have the condition

$$
N \frac{\left\langle\delta \beta_{\perp}^{2}\right\rangle \gamma^{2}}{1+n_{\perp}^{2} \gamma^{2}+\beta_{\perp}^{2} \gamma^{2}} \ll 1,
$$

where $\left\langle\delta \beta_{\perp}^{2}\right\rangle$ is a typical value of the variance of $\beta_{\perp}$ for a given particle wave packet.

Thus, in the scalar case,

$$
C\left(\mathbf{k}_{2}, \mathbf{k}_{1}\right)=\frac{e^{2}}{(2 \pi)^{3} 2 V k_{0}} c\left(\mathbf{k}_{2}\right) c^{*}\left(\mathbf{k}_{1}\right),
$$

where

$$
c(\mathbf{k}):=\int_{-\infty}^{\infty} d t e^{-i q_{i}\left[k_{0}-k_{\perp}^{2} /\left(2 P_{0 i}\right)\right] t+i q_{i} k_{3} x_{3}+i \mathbf{k}_{\perp} \mathbf{x}_{\perp}} q^{1 / 2}(\mathbf{f} \dot{\mathbf{x}}),
$$

and

$$
\dot{\mathbf{x}}(t)=\mathbf{P}(t) / P_{0}(t), \quad q(t)=P_{0}(t) / P_{0}^{\prime}(t),
$$

and $q_{i}=P_{0 i} / P_{0 i}^{\prime}$. Recall that $P_{0 i}$ is the energy of particle in the initial state and $P_{0 i}^{\prime}=P_{0 i}-k_{0}$. The approximate expression for probability (5) derived from (28) is nonnegative. Furthermore, matrix element (28) with the photon momenta satisfying (8) transforms properly under translations in the spacetime, $x^{\mu} \rightarrow x^{\mu}+a^{\mu}$, viz.,

$$
C\left(\mathbf{k}_{2}, \mathbf{k}_{1}\right) \rightarrow C\left(\mathbf{k}_{2}, \mathbf{k}_{1}\right) e^{i\left(\mathbf{k}_{2 \perp}-\mathbf{k}_{1 \perp}\right) \mathbf{a}_{\perp},}
$$

where $\mathbf{a}_{\perp}$ is the translation 4-vector component perpendicular to the detector axis.

Employing formula (A5), we find the leading contribution to the probability of radiation of a twisted photon by a charged scalar particle with the quantum recoil taken into account,

$$
\begin{aligned}
d P\left(s, m, k_{\perp}, k_{3}\right)= & e^{2} \mid \int_{-\infty}^{\infty} d t e^{-i q_{i}\left[k_{0}-k_{\perp}^{2} /\left(2 P_{0 i}\right)\right] t+i q_{i} k_{3} x_{3}} q^{1 / 2} \\
& \times\left.\left(\frac{1}{2}\left[\dot{x}_{+} a_{-}+\dot{x}_{-} a_{+}\right]+\dot{x}_{3} a_{3}\right)\right|^{2} \\
& \times\left(\frac{k_{\perp}}{2 k_{0}}\right)^{3} \frac{d k_{3} d k_{\perp}}{2 \pi^{2}}
\end{aligned}
$$

where

$a_{ \pm} \equiv a_{ \pm}\left(s, m, k_{3}, k_{\perp} ; \mathbf{x}\right), \quad a_{3} \equiv a_{3}\left(s, m, k_{\perp} ; \mathbf{x}\right)$.

Recall that we use the system of units such that $e^{2}=4 \pi \alpha$. In the case $q \approx 1$, i.e., when the energy of the radiated photon is negligibly smaller than the energy of charged particle, formula (32) passes into formula (36) in Ref. [33]. As a rule, $q \approx$ const in the ultrarelativistic limit, and so $q^{1 / 2}$ can be removed from the integrand of (32). Since $q>1$, we see that the quantum recoil tends to increase the radiation probability in comparison with the answer which does not include it.

\section{B. Dirac particle}

In case of radiation of twisted photons by the Dirac particles, the considerations are analogous but more cumbersome. Using the notation of Ref. [4], we have approximately

$$
\begin{aligned}
M\left(t_{1}, \mathbf{k}_{1}\right) & =\left(\frac{m_{e}}{P_{01}}\right)^{1 / 2} \bar{u}_{s^{\prime}}\left(\mathbf{P}_{1}\right) \hat{\mathbf{f}}_{1}^{*} e^{-i \mathbf{k}_{1} \mathbf{x}_{1}} u_{s}\left(\mathbf{P}_{1}\right)\left(\frac{m_{e}}{P_{01}}\right)^{1 / 2} \\
& =e^{-i \mathbf{k}_{1} \mathbf{x}_{1}}\left(\frac{m_{e}}{P_{01}^{\prime}}\right)^{1 / 2} \bar{u}_{s^{\prime}}\left(\mathbf{P}_{1}^{\prime}\right) \hat{\mathbf{f}}_{1}^{*} u_{s}\left(\mathbf{P}_{1}\right)\left(\frac{m_{e}}{P_{01}}\right)^{1 / 2}, \\
M^{\dagger}\left(t_{2}, \mathbf{k}_{2}\right) & =\left(\frac{m_{e}}{P_{02}}\right)^{1 / 2} \bar{u}_{s}\left(\mathbf{P}_{2}\right) \hat{\mathbf{f}}_{2} e^{i \mathbf{k}_{2} \mathbf{x}_{2}} u_{s^{\prime}}\left(\mathbf{P}_{2}\right)\left(\frac{m_{e}}{P_{02}}\right)^{1 / 2} \\
& =\left(\frac{m_{e}}{P_{02}}\right)^{1 / 2} \bar{u}_{s}\left(\mathbf{P}_{2}\right) \hat{\mathbf{f}}_{2} u_{s^{\prime}}\left(\mathbf{P}_{2}^{\prime}\right)\left(\frac{m_{e}}{P_{02}^{\prime}}\right)^{1 / 2} e^{i \mathbf{k}_{2} \mathbf{x}_{2}},
\end{aligned}
$$

where $s$ and $s^{\prime}$ characterize the initial and final electron spin states. For brevity, we do not write the hats over the operators $\mathbf{P}$ and $\mathbf{x}$ anymore. Further, we employ formulas (15) and (22), substitute all the operators by their averages, sum over spin polarizations of the escaping electron, and average over spin polarizations of the incoming electron. As a result, we come to 


$$
\frac{1}{2} \sum_{\text {spins }} M^{\dagger}\left(t_{2}, \mathbf{k}_{2}\right) M\left(t_{1}, \mathbf{k}_{1}\right) \rightarrow \frac{1}{2} S p\left[\left(A_{2}^{*}-i\left(\mathbf{B}_{2}^{*} \boldsymbol{\sigma}\right)\right)\left(A_{1}+i\left(\mathbf{B}_{1} \boldsymbol{\sigma}\right)\right)\right]=A_{2}^{*} A_{1}+\left(\mathbf{B}_{2}^{*} \mathbf{B}_{1}\right),
$$

where

$$
\begin{aligned}
& A_{1,2}=-\frac{\left(\mathbf{f}_{1,2}^{*} \mathbf{P}_{1,2}\right)}{2 \sqrt{P_{01,2}^{\prime} P_{01,2}}}\left(\sqrt{\frac{P_{01,2}^{\prime}+m_{e}}{P_{01,2}+m_{e}}}+\sqrt{\frac{P_{01,2}+m_{e}}{P_{01,2}^{\prime}+m_{e}}}\right), \\
& \mathbf{B}_{1,2}=-\frac{1}{2 \sqrt{P_{01,2}^{\prime} P_{01,2}}}\left(\sqrt{\frac{P_{01,2}^{\prime}+m_{e}}{P_{01,2}+m_{e}}}\left[\mathbf{f}_{1,2}^{*}, \mathbf{P}_{1,2}\right]-\sqrt{\frac{P_{01,2}+m_{e}}{P_{01,2}^{\prime}+m_{e}}}\left[\mathbf{f}_{1,2}^{*}, \mathbf{P}_{1,2}^{\prime}\right]\right) .
\end{aligned}
$$

In the ultrarelativistic limit, we can neglect the mass in expressions (36) since it gives the contributions of order $1 / \gamma$ as compared to the main contribution. Then,

$$
\begin{aligned}
& A_{2}^{*} A_{1}+\left(\mathbf{B}_{2}^{*} \mathbf{B}_{1}\right)=\frac{1}{4 P_{01}^{\prime} P_{02}^{\prime}}\left[\left(P_{01}+P_{01}^{\prime}\right)\left(P_{02}+P_{02}^{\prime}\right)\left(\mathbf{f}_{2} \dot{\mathbf{x}}_{2}\right)\left(\mathbf{f}_{1}^{*} \dot{\mathbf{x}}_{1}\right)\right. \\
& \left.-k_{0}^{2}\left(\mathbf{f}_{2}, \dot{\mathbf{x}}_{1}-\mathbf{n}_{1}\right)\left(\mathbf{f}_{1}^{*}, \dot{\mathbf{x}}_{2}-\mathbf{n}_{2}\right)+k_{0}^{2}\left(\mathbf{f}_{2} \mathbf{f}_{1}^{*}\right)\left(\dot{\mathbf{x}}_{2}-\mathbf{n}_{2}, \dot{\mathbf{x}}_{1}-\mathbf{n}_{1}\right)\right] .
\end{aligned}
$$

This expression turns into formula (2.41) in Ref. [4] for $\mathbf{k}_{1,2}=\mathbf{k}$.

The integration over azimuth angles of the vectors $\mathbf{k}_{1,2}$ is considered in Appendix C. With the aid of the notation introduced there, we can write

$$
\begin{aligned}
d P\left(s, m, k_{\perp}, k_{3}\right)= & e^{2} \int_{-\infty}^{\infty} \frac{d t_{1} d t_{2}}{4 P_{01}^{\prime} P_{02}^{\prime}} e^{-i\left(k_{0}-k_{\perp}^{2} /\left(2 P_{0 i}\right)\right) q_{i}\left(t_{2}-t_{1}\right)+i k_{3} q_{i}\left(x_{23}-x_{13}\right)} \\
& \times\left\{\left(P_{01}+P_{01}^{\prime}\right)\left(P_{02}+P_{02}^{\prime}\right)\left(\frac{1}{2}\left[\dot{x}_{1-} a_{-}^{*}+\dot{x}_{1+} a_{+}^{*}\right]+\dot{x}_{13} a_{3}^{*}\right)\left(\frac{1}{2}\left[\dot{x}_{2+} a_{-}+\dot{x}_{2-} a_{+}\right]+\dot{x}_{23} a_{3}\right)\right. \\
& +\frac{k_{0}^{2}}{4}\left[\left(\dot{x}_{1+} a_{+}^{*}-i n_{\perp} a_{+}^{*}(m-1)\right)\left(\dot{x}_{2-} a_{+}+i n_{\perp} a_{+}(m-1)\right)\right. \\
& \left.\left.+\left(\dot{x}_{1-} a_{-}^{*}+i n_{\perp} a_{-}^{*}(m+1)\right)\left(\dot{x}_{2+} a_{-}-i n_{\perp} a_{-}(m+1)\right)\right]\right\}\left(\frac{k_{\perp}}{2 k_{0}}\right)^{3} \frac{d k_{3} d k_{\perp}}{2 \pi^{2}}
\end{aligned}
$$

in the leading order in $\varkappa / \gamma$. Here,

$$
\begin{array}{ll}
a_{ \pm} \equiv a_{ \pm}\left(s, m, k_{3}, k_{\perp} ; \mathbf{x}_{2}\right), & a_{3} \equiv a_{3}\left(s, m, k_{\perp} ; \mathbf{x}_{2}\right), \\
a_{ \pm}^{*} \equiv a_{ \pm}^{*}\left(s, m, k_{3}, k_{\perp} ; \mathbf{x}_{1}\right), & a_{3}^{*} \equiv a_{3}^{*}\left(s, m, k_{\perp} ; \mathbf{x}_{1}\right) .
\end{array}
$$

The approximate expression (38) for probability density (5) is non-negative. In the case $k_{0} \ll \varepsilon_{1,2}$, formula (38) passes into expression (36) in Ref. [33] without the quantum recoil due to photon radiation. Just as for the scalar particle case, we see that the quantum recoil tends to increase the radiation probability as compared to the formula without recoil. As long as

$$
\frac{P_{0}+P_{0}^{\prime}}{2 P_{0}^{\prime}}=\frac{1+q}{2}, \quad \frac{k_{0}}{P_{0}^{\prime}}=q-1=: \delta q,
$$

the second and third terms in (38) are proportional to $(\delta q)^{2}$, while the first term is proportional to $(1+\delta q / 2)^{2}$. Thus, in the limit of small recoil, $q \approx 1$, the second and third terms can be neglected, and the contribution of the first term is the same as in the case of a scalar particle (32) since $q^{1 / 2} \approx 1+\delta q / 2$. The spin effects become irrelevant in this limit within the bounds of the approximations made in deriving (38).

Contrary to the classical formula for radiation probability, expression (38) does not factorize into $c^{*} c$, where $c$ is determined by the particle trajectory. This is a consequence of the fact that the averaging over spin polarizations was performed and the quantum recoil was taken into account. If one neglects the recoil, the created radiation will be described by a coherent state in the Fock space [33,69,85-87], and nontrivial quantum correlations will be absent. In the case of a scalar charged particle, formula (32) does factorize into the amplitude and its complex conjugate, and the nontrivial quantum correlations are absent within the bounds of the approximations made.

Notice that formulas (32) and (38) describe the radiation produced by one charged particle. As a rule, in real experiments, the bunch of charged particles radiates. Under usual conditions, radiation amplitudes of hard photons produced 
by different particles in the bunch add up incoherently; i.e., expression (32) or (38) should be summed over different particles in the bunch. In Refs. [77,78], the simple formulas were obtained, allowing one to find the radiation of twisted photons by a bunch of charged particles using the oneparticle radiation probability distribution. These formulas can be applied to (32) and (38), as the initial plane wave matrix elements (31) transform correctly under translations. Below, we shall employ the formulas from Ref. [77] for axially symmetric bunches. Such bunches are created, for example, in the electron-positron collider VEPP-2000, Novosibirsk [88].

\section{FORWARD RADIATION BY CHARGED PARTICLES IN UNDULATORS}

Let us apply the general formulas derived in the preceding section to the forward radiation of charged particles in undulators with the quantum recoil taken into account. We shall investigate the undulator radiation in the dipole regime for an arbitrary periodic trajectory of a charged particle. As for the wiggler case, we shall obtain the exact expression for the probability of radiation of twisted photons by charged particles moving along an ideal helix (the helical wiggler).

\section{A. Scalar particle}

The general formula for the radiation probability of a twisted photon by a scalar charged particle has the form (32). The radiation of twisted photons by undulators without quantum recoil was described in Ref. [33] (Sec. V). Comparing Eq. (82) in Ref. [33] with (32), we see that the account for quantum recoil leads only to a change of the energy spectrum of radiated photons and to the appearance of the common factor $q$ when the forward radiation produced by a scalar particle in the undulator in the dipole regime and in the ideal helical wiggler is considered. Notice that, according to the Lorentz equations,

$$
q=q_{i}=\mathrm{const}
$$

for the motion of charged particles in undulators. As a result, in the dipole approximation,

$$
\begin{aligned}
d P\left(s, m, k_{\perp}, k_{3}\right)= & e^{2} n_{\perp}^{3} \sum_{n=1}^{\infty} \delta_{N}^{2}\left[q k_{0}\left(1-n_{3} v_{3}-\frac{n_{\perp} k_{\perp}}{2 P_{0}}\right)-n \omega\right] \\
& \times q\left\{\delta_{m, 1}\left(k_{\perp} v_{3}+\frac{\omega n n_{\perp}}{n_{3}-s}\right)^{2}\left|r_{+}(n)\right|^{2}+\delta_{m,-1}\left(k_{\perp} v_{3}+\frac{\omega n n_{\perp}}{n_{3}+s}\right)^{2}\left|r_{-}(n)\right|^{2}\right\} \frac{d k_{3} d k_{\perp}}{16},
\end{aligned}
$$

where the notation introduced in Ref. [33] was used. As in the case of radiation without recoil, the main part of forward radiation consists of twisted photons with $m= \pm 1$.

The energy spectrum is found from the equation

$$
P_{0} k_{0}^{n}\left(1-n_{3} v_{3}-\frac{n_{\perp}^{2} k_{0}^{n}}{2 P_{0}}\right)=n \omega\left(P_{0}-k_{0}^{n}\right) .
$$

In virtue of condition (17) with minimum $\sigma_{\perp}$ taken from (B6), the last term in the parentheses on the left-hand side is small and should be taken into account perturbatively. Indeed,

$$
1-n_{3} v_{3}-\frac{n_{\perp}^{2} k_{0}^{n}}{2 P_{0}} \approx \frac{1+K^{2}+n_{\perp}^{2} \gamma^{2}-n_{\perp}^{2} \gamma^{2} k_{0}^{n} / P_{0}}{2 \gamma^{2}}=\frac{1+K^{2}+n_{\perp}^{2} \gamma^{2} / q}{2 \gamma^{2}}
$$

For $k_{0} \sim \varepsilon$, it follows from (17) and (B6) that $n_{\perp} \gamma \ll K$, and the contribution of this term can be neglected as compared to the contributions of the first terms. For $k_{0} \ll \varepsilon$, obviously, the contribution of this term is also negligibly small in comparison with the contributions of the first terms in this expression. Then, the physical solution to (43) takes the form

$$
\begin{aligned}
k_{0}^{n} & =\frac{P_{0}\left(1-n_{3} v_{3}\right)}{n_{\perp}^{2}}\left\{1+\frac{\bar{k}_{0}^{n}}{P_{0}}-\left[\left(1+\frac{\bar{k}_{0}^{n}}{P_{0}}\right)^{2}-\frac{2 \bar{k}_{0}^{n} n_{\perp}^{2}}{P_{0}\left(1-n_{3} v_{3}\right)}\right]^{1 / 2}\right\} \\
& \approx \frac{P_{0}\left(1+K^{2}+n_{\perp}^{2} \gamma^{2}\right)}{2 n_{\perp}^{2} \gamma^{2}}\left\{1+\frac{\bar{k}_{0}^{n}}{P_{0}}-\left[\left(1+\frac{\bar{k}_{0}^{n}}{P_{0}}\right)^{2}-\frac{4 \bar{k}_{0}^{n} n_{\perp}^{2} \gamma^{2}}{P_{0}\left(1+K^{2}+n_{\perp}^{2} \gamma^{2}\right)}\right]^{1 / 2}\right\},
\end{aligned}
$$

where

$$
\bar{k}_{0}^{n}:=\frac{\omega n}{1-n_{3} v_{3}}
$$


is the energy of the radiated twisted photon without quantum recoil. If condition (17) with minimum $\sigma_{\perp}$ taken from (B6) is fulfilled, the last term under the square root in (45) is small. Developing (45) as a series, we obtain in the leading order

$$
k_{0}^{n}=\frac{n \omega}{1-n_{3} v_{3}+n \omega / P_{0}}=\frac{\bar{k}_{0}^{n}}{1+\bar{k}_{0}^{n} / P_{0}} \Leftrightarrow \frac{1}{k_{0}^{n}}=\frac{1}{\bar{k}_{0}^{n}}+\frac{1}{P_{0}} .
$$

In fact, this is the BK prescription for the shift of the energy spectrum due to quantum recoil. Formulas (45) and (47) imply that $k_{0}^{n}<P_{0} \approx \varepsilon$.

Using formulas from Ref. [33], the probability of radiation of twisted photons by a scalar charged particle moving along an ideal helix can readily be derived even in the wiggler regime. Denoting by $\chi= \pm 1$ the helicity of the particle trajectory, we find from (32) that

$$
\begin{aligned}
d P\left(s, m, k_{\perp}, k_{3}\right)= & e^{2} n_{\perp}^{3} \delta_{N}^{2}\left[q k_{0}\left(1-n_{3} v_{3}-\frac{n_{\perp} k_{\perp}}{2 P_{0}}\right)-\chi m \omega\right] \\
& \times q\left[\left(v_{3}-\chi \frac{n_{3} \omega m}{n_{\perp} k_{\perp}}\right) J_{m}\left(\frac{k_{\perp} K}{\omega \gamma}\right)-\chi \frac{s K}{n_{\perp} \gamma} J_{m}^{\prime}\left(\frac{k_{\perp} K}{\omega \gamma}\right)\right]^{2} \frac{d k_{3} d k_{\perp}}{4} .
\end{aligned}
$$

Thus, the selection rule $m=\chi n$ for the forward radiation produced by an ideal helical wiggler survives even when the quantum recoil is taken into account, within the bounds of the approximations made in deriving formula (32). The energy spectrum of radiated twisted photons is given by (45) and (47). Notice that formula (48) is the exact one. In contrast to expression (42), its domain of applicability is not restricted to the dipole regime. The particular case of formula (42) can be reproduced from (48) by expanding the latter in a Taylor series in $K$.

\section{B. Dirac particle}

In the dipole approximation, it is necessary to expand the integrand of (38) into series in $K$ and take into account only the leading contribution (see the estimates in Sec. 5. A of Ref. [33]). Then, up to a common factor, the first term in the curly brackets in (38) turns into the same expression as that appearing when the quantum recoil is neglected,

$$
\begin{gathered}
\frac{1}{4}\left(P_{0}+P_{0}^{\prime}\right)^{2}\left[-\delta_{m, 1}\left(\frac{i n_{\perp}}{n_{3}-s} \dot{r}_{2+}+k_{\perp} v_{3} r_{2+}\right)\left(\frac{i n_{\perp}}{n_{3}-s} \dot{r}_{1-}+k_{\perp} v_{3} r_{1-}\right)\right. \\
\left.-\delta_{m,-1}\left(\frac{i n_{\perp}}{s+n_{3}} \dot{r}_{2-}-k_{\perp} v_{3} r_{2-}\right)\left(\frac{i n_{\perp}}{s+n_{3}} \dot{r}_{1+}+k_{\perp} v_{3} r_{1+}\right)\right] .
\end{gathered}
$$

The second term in the curly brackets in (38) standing at $k_{0}^{2} / 4$ is written as

$$
\begin{aligned}
& \frac{n_{\perp}^{2}}{\left(s+n_{3}\right)^{2}}\left[\dot{r}_{1+} \delta_{m,-1}-i n_{\perp}\left(\delta_{m, 0}+\frac{1}{2} \delta_{m, 1} k_{\perp} r_{1-}-\frac{1}{2} \delta_{m,-1} k_{\perp} r_{1+}\right)\right] \\
& \quad \times\left[\dot{r}_{2-} \delta_{m,-1}+i n_{\perp}\left(\delta_{m, 0}+\frac{1}{2} \delta_{m, 1} k_{\perp} r_{2+}-\frac{1}{2} \delta_{m,-1} k_{\perp} r_{2-}\right)\right] \\
& \quad+\frac{n_{\perp}^{2}}{\left(s-n_{3}\right)^{2}}\left[\dot{r}_{1-} \delta_{m, 1}+i n_{\perp}\left(\delta_{m, 0}+\frac{1}{2} \delta_{m, 1} k_{\perp} r_{1-}-\frac{1}{2} \delta_{m,-1} k_{\perp} r_{1+}\right)\right] \\
& \quad \times\left[\dot{r}_{2+} \delta_{m, 1}-i n_{\perp}\left(\delta_{m, 0}+\frac{1}{2} \delta_{m, 1} k_{\perp} r_{2+}-\frac{1}{2} \delta_{m,-1} k_{\perp} r_{2-}\right)\right] .
\end{aligned}
$$

Expanding $r_{1,2}$ into a Fourier series and integrating over the time variable, we have

$$
\begin{aligned}
d P\left(s, m, k_{\perp}, k_{3}\right)= & e^{2} n_{\perp}^{3} \sum_{n=1}^{\infty} \delta_{N}^{2}\left[q k_{0}\left(1-n_{3} v_{3}-\frac{n_{\perp} k_{\perp}}{2 P_{0}}\right)-n \omega\right] \\
& \times\left\{\delta_{m, 1}\left[\left(P_{0}+P_{0}^{\prime}\right)^{2}\left(k_{\perp} v_{3}+\frac{\omega n n_{\perp}}{n_{3}-s}\right)^{2}+\frac{k_{\perp}^{2}}{\left(n_{3}-s\right)^{2}}\left(\omega n-\frac{n_{\perp} k_{\perp}}{2}\right)^{2}+\frac{n_{\perp}^{2} k_{\perp}^{4}}{4\left(n_{3}+s\right)^{2}}\right]\left|r_{+}(n)\right|^{2}\right. \\
& \left.+\delta_{m,-1}\left[\left(P_{0}+P_{0}^{\prime}\right)^{2}\left(k_{\perp} v_{3}+\frac{\omega n n_{\perp}}{n_{3}+s}\right)^{2}+\frac{k_{\perp}^{2}}{\left(n_{3}+s\right)^{2}}\left(\omega n-\frac{n_{\perp} k_{\perp}}{2}\right)^{2}+\frac{n_{\perp}^{2} k_{\perp}^{4}}{4\left(n_{3}-s\right)^{2}}\right]\left|r_{-}(n)\right|^{2}\right\} \frac{d k_{3} d k_{\perp}}{64 P_{0}^{\prime 2}} .
\end{aligned}
$$



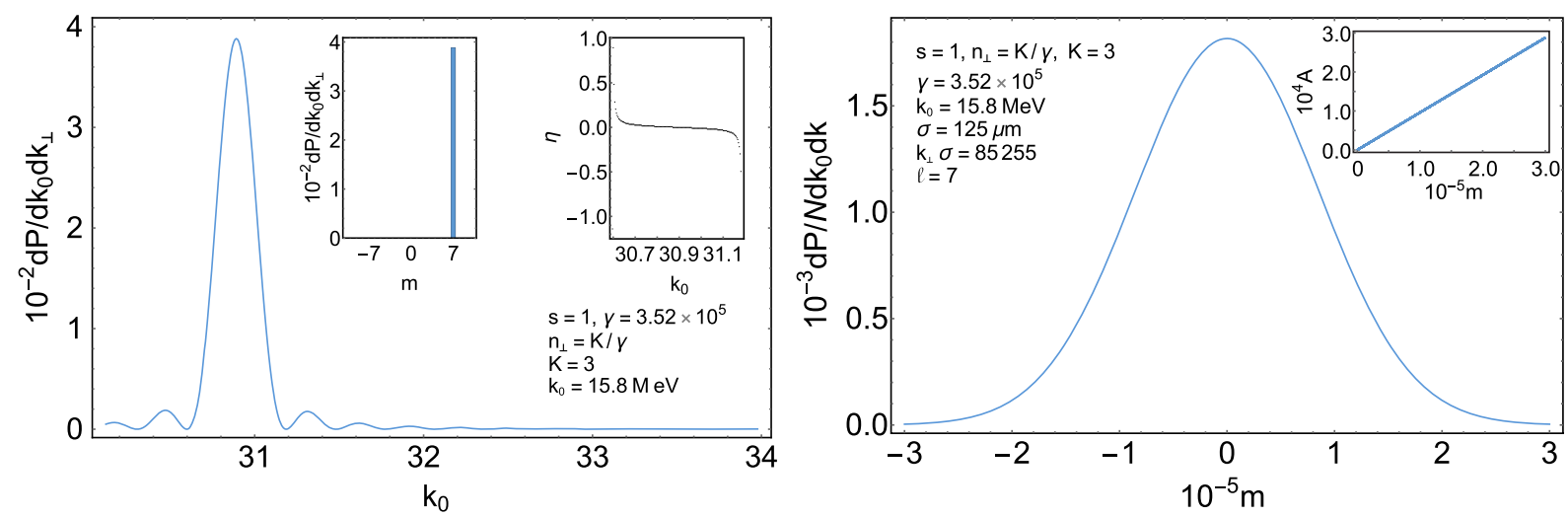

FIG. 1. The radiation of twisted photons by $180 \mathrm{GeV}$ electrons in the helical wiggler. The seventh harmonic is presented. The wiggler period is $0.72 \mathrm{~cm}$, the number of periods $N=15$, and the magnetic field strength in the wiggler is $63.3 \mathrm{kG}$. The applicability conditions (84) are satisfied for $\sigma_{\perp} \lesssim 10^{3} \mathrm{~m}_{e}^{-1}$. The photon energy is measured in the electron rest energies. Left panel: The probability of recording a twisted photon produced by one electron in the wiggler against the photon energy. Left inset: The probability distribution over $m$. Right inset: The relative change of radiation probability due to quantum recoil: $\eta:=\left(d P_{c l}-d P\right) / d P_{c l}$, where $d P_{c l}$ is the radiation probability without quantum recoil. Right panel: The distribution over $m$ of the probability per particle to record a twisted photon produced by an incoherent axially symmetric bunch of particles in the wiggler. The radial bunch profile is Gaussian with the width $\sigma=125 \mu \mathrm{m}$. The angular momentum per photon is the same as for radiation of one electron. Inset: The asymmetry of distribution over $m$.

As in the case of a scalar particle, only the twisted photons with $m= \pm 1$ are radiated. The photon energy spectrum has the form of (45) and (47).

Performing calculations along the same lines as in the case of a scalar particle, we find for an ideal helical wiggler the exact result

$$
\begin{aligned}
d P\left(s, m, k_{\perp}, k_{3}\right)= & e^{2} n_{\perp}^{3} \delta_{N}^{2}\left[q k_{0}\left(1-n_{3} v_{3}-\frac{n_{\perp} k_{\perp}}{2 P_{0}}\right)-\chi m \omega\right] \\
& \times\left\{\left(P_{0}+P_{0}^{\prime}\right)^{2}\left[\left(v_{3}-\chi \frac{n_{3} \omega m}{n_{\perp} k_{\perp}}\right) J_{m}-\chi \frac{s K}{n_{\perp} \gamma} J_{m}^{\prime}\right]^{2}+\frac{k_{\perp}^{2}}{4\left(n_{3}+s\right)^{2}}\left(\frac{K}{\gamma} J_{m+1}-\chi n_{\perp} J_{m}\right)^{2}\right. \\
& \left.+\frac{k_{\perp}^{2}}{4\left(n_{3}-s\right)^{2}}\left(\frac{K}{\gamma} J_{m-1}-\chi n_{\perp} J_{m}\right)^{2}\right\} \frac{d k_{3} d k_{\perp}}{16 P_{0}^{\prime 2}} .
\end{aligned}
$$

The argument of the Bessel functions is the same as in formula (48). Employing the recurrence relations for the Bessel functions and keeping in mind that $n_{3} \approx 1$, the last two terms in the curly brackets can be brought to

$$
k_{0}^{2}\left[\left(\frac{\omega m}{n_{\perp} k_{\perp}}-1\right) J_{m}+\frac{s K}{n_{\perp} \gamma} J_{m}^{\prime}\right]^{2},
$$

to the accuracy with which we work. Setting $v_{3}=n_{3}=1$ in the first term in the curly brackets in (52), we obtain

$$
\begin{aligned}
d P\left(s, m, k_{\perp}, k_{3}\right) \approx & e^{2} n_{\perp}^{3} \delta_{N}^{2}\left[q k_{0}\left(1-n_{3} v_{3}-\frac{n_{\perp} k_{\perp}}{2 P_{0}}\right)-\chi m \omega\right] \\
& \times\left(1+q^{2}\right)\left[\left(1-\chi \frac{\omega m}{n_{\perp} k_{\perp}}\right) J_{m}-\chi \frac{s K}{n_{\perp} \gamma} J_{m}^{\prime}\right]^{2} \\
& \times \frac{d k_{3} d k_{\perp}}{8} .
\end{aligned}
$$

The forward radiation of twisted photons in helical wigglers obeys the selection rule $m=\chi n$ within the bounds of the approximations made (see Fig. 1). Comparing (48) with (54), we see that the probability of radiation of twisted photons by Dirac particles is always bigger than the same quantity for scalar particles since $\left(1+q^{2}\right) / 2>q$ for $q>1$ (see Fig. 2). For $q \approx 1$, the respective radiation probabilities are almost equal. Of course, developing (54) as a Taylor series in $K$, one reproduces the particular case of (51).

\section{Number of radiated twisted photons}

Let us find the number of radiated twisted photons with a given projection of the total angular momentum $m$ per energy interval $k_{0}$ and the total number of radiated twisted photons. Further, we set $\chi=1$, since $\chi=-1$ can be obtained by the substitution $m \rightarrow-m, s \rightarrow-s$. To shorten formulas, we suppose that the last term in the round brackets in (43) is small and the energy spectrum is given by (47). Then, 

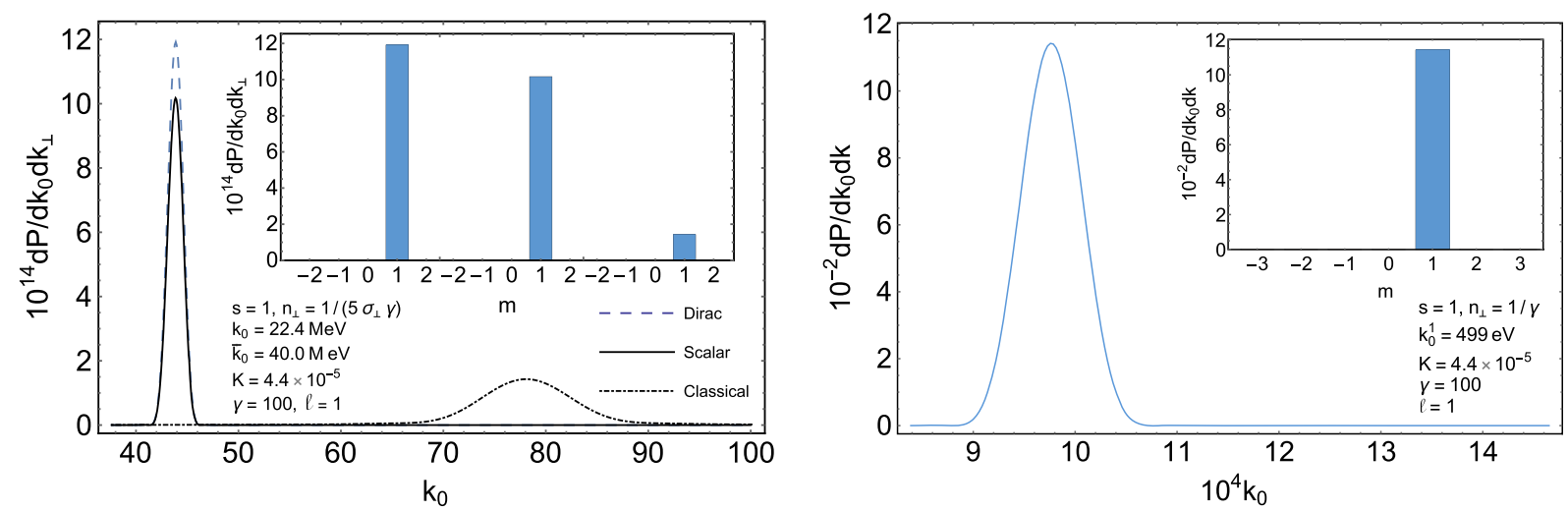

FIG. 2. The radiation of twisted photons by $51.1 \mathrm{MeV}$ electrons evolving in the circularly polarized electromagnetic wave produced by the FEL with the photon energy $1 \mathrm{keV}$, intensity $3.38 \times 10^{15} \mathrm{~W} / \mathrm{cm}^{2}$, and amplitude envelope (163) with $N=20$. These data correspond to $\Omega \approx 1.96 \times 10^{-3}$ and $f_{0} \approx 8.54 \times 10^{-8}$. The applicability conditions (84) are satisfied for $\sigma_{\perp} \lesssim 10^{3} m_{e}^{-1}$. The photon energy is measured in the electron rest energies. Left panel: The head-on collision. The first harmonic (166) for radiation without recoil (classical), scalar, and Dirac particles is depicted. For the parameters chosen, the quantum recoil halves the energy of radiated photons in comparison with formula without recoil. The probability of radiation of twisted photons by Dirac particles is bigger than the same quantity for scalar particles, which, in turn, is bigger than the probability of radiation of twisted photons without quantum recoil [see the discussion after Eq. (54)]. Right panel: The laser wave is overtaking the electron. The first harmonic (166) is shown. The quantum recoil is negligible in this case. Insets: The distribution over $m$ at the main maximum of the harmonic.

$$
\begin{aligned}
& \delta_{N}^{2}\left[q k_{0}\left(1-n_{3} v_{3}\right)-m \omega\right] d k_{3} d k_{\perp} \\
& \quad=\delta_{N}^{2}(x) \frac{d k_{0} d x}{v_{3} q n_{\perp} \omega} \approx \delta_{N}^{2}(x) \frac{d k_{0} d x}{q n_{\perp} \omega} .
\end{aligned}
$$

As long as the function $\delta_{N}^{2}(x)$ is localized near the point $x=0$ for $N \gtrsim 5$, the integral over $x$ can easily be found:

$$
\int_{-\infty}^{\infty} d x \delta_{N}^{2}(x)=N
$$

Substituting (55) and (56) into (48) and (54), we obtain $d P\left(s, m, k_{0}\right) / d k_{0}$. The analogous formulas can be obtained in the dipole case as well. However, in that case, the overlapping of harmonics has to be taken into account. Namely, the integration over $x$ results in

$$
\begin{aligned}
& \sum_{n=1}^{\infty} \delta_{N}^{2}\left[q k_{0}\left(1-n_{3} v_{3}\right)-n \omega\right] d k_{3} d k_{\perp} \\
& \quad \rightarrow N \sum_{n=1}^{\infty} \theta_{k_{0}}\left[\frac{n \omega}{1+n \omega / P_{0}}, \frac{n \omega}{1-v_{3}+n \omega / P_{0}}\right] \frac{d k_{0}}{q n_{\perp} \omega},
\end{aligned}
$$

where

$$
\theta_{x}[a, b]:= \begin{cases}1, & x \in[a, b] \\ 0, & x \notin[a, b],\end{cases}
$$

and $n_{\perp}$ is to be expressed through $k_{0}^{n}$ by using (47).

The twisted photons with large projections $m$ of the total angular momentum can be produced by undulators only in the wiggler regime. When $m \gtrsim 5$, the Bessel functions entering into (48) and (54) are expressed through the Airy functions [9] with (see the notation in Eq. (122) of Ref. [33])

$$
x \approx 1-\frac{4 n_{\perp}^{2} \gamma^{2} K^{2}}{\left(1+K^{2}+n_{\perp}^{2} \gamma^{2}\right)^{2} q^{2}}
$$

For the probability of radiation of twisted photons with the total angular momentum projection $m, m \gtrsim 5$, not to be exponentially suppressed, quantity (59) must be small. This is achieved when $K \gtrsim 3$ and

$$
n_{k}:=n_{\perp} \gamma / K \approx 1
$$

Then, it follows from (54), (55), and (56) that

$$
\begin{aligned}
\frac{d P\left(s, m, k_{0}\right)}{d k_{0}} \approx & \pi N \alpha \frac{K^{2}}{\gamma^{2}} \frac{q+q^{-1}}{2 \omega}\left(\frac{2}{m}\right)^{4 / 3}\left[\operatorname{Ai}^{\prime}(y)\right. \\
& \left.+s_{k}\left(1-q \frac{1+n_{k}^{2}}{2 n_{k}^{2}}\right)\left(\frac{m}{2}\right)^{1 / 3} \operatorname{Ai}(y)\right]^{2},
\end{aligned}
$$

where

$$
y \approx\left(\frac{m}{2}\right)^{2 / 3}\left[1-\frac{4 n_{k}^{2}}{\left(1+n_{k}^{2}\right)^{2} q^{2}}\right]
$$

and $n_{k}$ should be expressed in terms of $k_{0}^{m}$ from (47) and (60). The Airy function and its derivative drop exponentially to zero for $y \gtrsim 1 / 2$. Therefore, the radiation of twisted photons is exponentially suppressed when 


$$
m \gtrsim m_{c}, \quad m_{c}:=\min \left(\left(1-q^{-2}\right)^{-3 / 2}, K^{3}\right) / \sqrt{2} .
$$

The second quantity in the min function appearing in the definition of $m_{c}$ comes from the estimate of subsequent terms of the expansion of $y$ in $K^{-1}$ (see Eq. (120) of Ref. [33]).

The distribution (61) reaches the maximum at

$$
n_{k} \approx q-s c_{0}(2 / m)^{1 / 3},
$$

where $c_{0} \approx 0.58$ is found from the equation

$$
\left(\operatorname{Ai}^{\prime}\left(c_{0}^{2}\right)-c_{0} \operatorname{Ai}\left(c_{0}^{2}\right)\right)^{\prime}=0 .
$$

If $m<m_{c}$, then

$$
\frac{d P\left(m, k_{0}\right)}{d k_{0}}=\sum_{s= \pm 1} \frac{d P\left(s, m, k_{0}\right)}{d k_{0}}
$$

possesses the maxima at points (64) with $s= \pm 1$ and the local minimum at the point $n_{k} \approx q$. For $m \gtrsim m_{c}$, the maxima coalesce in the point $n_{k} \approx q$.

Such behavior of radiation maxima is expectable, if one bears in mind that the wiggler radiation is just the synchrotron one in the Lorentz frame in which the electron is at rest on average. The intensity profiles of lower synchrotron harmonics, $m \ll m_{c}$, were thoroughly investigated in Sec. 1.3.4 of Ref. [9], in which the effect of a blossoming out rose was revealed. The maxima of intensity of these harmonics do not lie in the orbit plane in the ultrarelativistic limit. In the orbit plane, the intensity of these harmonics possesses a local minimum. For large harmonics, $m \gtrsim m_{c}$, this minimum disappears. The maxima and minima of intensities of synchrotron harmonics in the laboratory frame are found from the standard transformation law for angles

$\sin \theta=\frac{\sin \theta^{\prime}}{1+\left[1-\left(1+K^{2}\right) / \gamma^{2}\right]^{1 / 2} \cos \theta^{\prime}} \frac{\sqrt{1+K^{2}}}{\gamma}$,

where $\theta^{\prime}$ is the polar angle counted in the "synchrotron" frame and $\theta$ is the same angle in the laboratory frame. In the synchrotron frame, the electron has the Lorentz factor

$$
\gamma_{s}=\sqrt{1+K^{2}}
$$

and is ultrarelativistic in the wiggler case. The orbit plane, $\theta^{\prime}=\pi / 2$, is seen in the laboratory frame at the angle $\theta=\arcsin \left(\sqrt{1+K^{2}} / \gamma\right) \approx K / \gamma$. We shall return to the effect of the blossoming out rose in Sec. IV.

Now, we find a loose estimate for the number of twisted photons radiated by the right-handed helical wiggler at the harmonic $n=m$. The function (61) is peaked at $n_{k} \approx 1$. The width of this peak can be found from the equation

$$
1-\frac{4 n_{k}^{2}}{\left(1+n_{k}^{2}\right)^{2} q^{2}}=b\left(\frac{2}{m}\right)^{2 / 3}
$$

where $b \sim 1$. Solving this equation, we obtain

$$
\Delta n_{k} \approx 2 q\left[b(2 / m)^{2 / 3}+q^{-2}-1\right]^{1 / 2} .
$$

Take into account that

$$
d k_{0}=-\frac{v_{3}}{n_{3}} \frac{k_{0}^{2}}{\omega m} \frac{K^{2}}{\gamma^{2}} n_{k} d n_{k} .
$$

Then, assuming $(q-1) m^{1 / 3} \ll 1$ and multiplying the value of $d P / d n_{k}$ at the point $n_{k}=1$ by $\Delta n_{k}$, we arrive at

$$
\begin{aligned}
\Delta P(s, m) \approx & 4 \pi \alpha N(2 / m)^{1 / 3}\left[b(2 / m)^{2 / 3}+q^{-2}-1\right]^{1 / 2} \\
& \times \operatorname{Ai}^{2}\left(\left(\frac{m}{2}\right)^{2 / 3}\left(1-q^{-2}\right)\right) .
\end{aligned}
$$

This quantity is independent of $K$. In the classical limit, $q=1$, we have

$$
\Delta P(s, m) \approx 1.1 \times 10^{-2} \mathrm{Nm}^{-2 / 3},
$$

for $b=1.3$ [in Eq. (135) of Ref. [33], the other quantity was estimated]. Such a value of $b$ is taken for concordance of the estimate with the numerical calculations. This estimate shows, in particular, that, in describing the leading contribution to radiation of twisted photons produced in wigglers, the one-photon approximation is justified when

$$
N m^{-2 / 3} \lesssim 10 .
$$

If this condition is violated, the trajectory can be partitioned into pieces such that condition (74) is satisfied for each part of the trajectory. The probabilities of radiation from different parts of the trajectory should be summed and a change of the electron energy momentum due to the radiation reaction on the each part of the trajectory is to be taken into account. In the classical regime, $q-1 \ll 1$, the LandauLifshitz equation can be employed to describe the effective electron dynamics [15,19-21,34,35,89-97].

\section{Applicability conditions.}

Let us find the domain of applicability of the above formulas for the radiation of twisted photons in undulators with the quantum recoil taken into account. To shorten formulas, we suppose that all the dimensional quantities are measured in the units of the electron rest energy or in the electron Compton wavelengths.

Formula (63) implies that the quantum recoil diminishes the maximum attainable value of $m$ for the twisted photons generated in the forward undulator radiation. Another restriction on the maximum $m$ follows from the requirement (17). If 


$$
n_{\perp} \gamma \sigma_{\perp} \ll 1
$$

then (17) holds. The condition (75) can be satisfied only in the dipole regime. In the wiggler case, the main part of radiation is produced with $n_{\perp} \gamma \approx K \gtrsim 3$ and Eq. (75) is not fulfilled even for the wave packet with cross-size (B6).

If

$$
n_{\perp} \gamma \sigma_{\perp} \gtrsim 1
$$

then (17) and (47) imply

$$
\bar{k}_{0}^{m} / \gamma=q-1 \lesssim 1 /\left(10 n_{\perp} \gamma \sigma_{\perp}\right) \Rightarrow q-1 \ll 1 ;
$$

i.e., in radiating a photon, the quantum recoil experienced by the electron should be small. In the wiggler case, for $n_{k} \approx 1$, we obtain

$$
m \lesssim K /\left(10 \omega \gamma \sigma_{\perp}\right)=R /\left(10 \sigma_{\perp}\right),
$$

where $K=\omega \gamma R$ and $R$ is the radius of the spiral turn along which the electron is moving. Taking into account (B6), we deduce the upper estimate

$$
m \lesssim R K / 10
$$

where recall that $R$ is measured in the Compton wavelengths $\chi_{C}$. The estimates (78), (79) are the necessary conditions for the model of a point particle to be used. In this case, the localized wave packet of a particle radiating twisted photons in a wiggler can be characterized only by the average coordinate and momentum. As we see, the quantum recoil should be small for that to be the case.

It is useful to write restrictions (63) and (78) as a system of inequalities specifying the admissible region on the plane $\left(k_{0}^{m} / \varepsilon, m\right)$ :

$\frac{k_{0}^{m}}{\gamma} \lesssim \frac{1}{4}\left(\frac{2}{m}\right)^{2 / 3}, \quad m \lesssim \frac{K^{3}}{\sqrt{2}}, \quad \frac{k_{0}^{m}}{\gamma} \lesssim \frac{1}{10 K \sigma_{\perp}}$.

Note that $k_{0}^{m} \approx \bar{k}_{0}^{m}$. One can distinguish two cases:

$$
\text { i) } 5 \sigma_{\perp}>K, \quad \text { ii) } 5 \sigma_{\perp}<K \text {. }
$$

In case $\mathrm{i}$, the region (80) is reduced to a rectangle,

$$
\frac{k_{0}^{m}}{\gamma} \lesssim \frac{1}{10 K \sigma_{\perp}}, \quad m \lesssim \frac{K^{3}}{\sqrt{2}}
$$

The twisted photons with the maximum energy and projection of the total angular momentum are produced when inequalities (82) turn into the equalities. In case ii, the region (80) has nontrivial angular points at

$$
\begin{aligned}
& k_{0}^{m}=\frac{\gamma}{10 K \sigma_{\perp}}, \quad m=\frac{\left(5 K \sigma_{\perp}\right)^{3 / 2}}{\sqrt{2}} ; \\
& k_{0}^{m}=\frac{\gamma}{2 K^{2}}, \quad m=\frac{K^{3}}{\sqrt{2}},
\end{aligned}
$$

which coalesce for $5 \sigma_{\perp}=K$. In terms of $m$, the three inequalities in (80) are written as

$m \lesssim \frac{1}{2}\left(\frac{K^{2}}{\gamma \omega}\right)^{3 / 5}, \quad m \lesssim \frac{K^{3}}{\sqrt{2}}, \quad m \lesssim \frac{K}{10 \sigma_{\perp} \gamma \omega}$,

respectively.

\section{RADIATION BY CHARGED PARTICLES IN THE LASER WAVE}

Let us apply the above general formulas for a description of radiation of twisted photons by an ultrarelativistic charged particle in the laser wave of a circular polarization. We suppose that the one-photon radiation gives the leading contribution to radiation of twisted photons. Then, we can employ the formulas from the preceding sections to describe this radiation. The strength tensor of the electromagnetic field reads as (we use the notation borrowed from Refs. $[94,95,98])$

$$
e F^{\mu \nu}=f(\xi) h_{-}^{[\mu}\left[h_{1}^{\nu]} \cos \varphi(\xi)+h_{2}^{\nu]} \sin \varphi(\xi)\right]
$$

where $h_{-}^{\mu}=(1,0,0, \zeta), h_{1,2}^{\mu}=\delta_{1,2}^{\mu}$, the function $f(\xi)$ characterizes the amplitude of the electromagnetic field, and $\varphi(\xi)$ is the phase, where $\xi=h_{-}^{\mu} x_{\mu}=x^{0}-\zeta x^{3}$. We consider the situation in which the electromagnetic wave propagates along the axis of the detector of twisted photons. The quantity $\zeta= \pm 1$, where the upper sign corresponds to the case in which the wave moves toward the detector and the lower sign is for the case in which the wave moves from the detector.

It is useful to convert all the quantities to the dimensionless ones using the Compton wavelength as a unit length. Then, for example, the laser wave with intensity $10^{22} \mathrm{~W} / \mathrm{cm}^{2}$ and photon energy $1.53 \mathrm{eV}$ [99] corresponds to

$$
|f| \approx 1.47 \times 10^{-4}, \quad|\Omega| \approx 2.99 \times 10^{-6} .
$$

The Lorentz equations are easily solved for arbitrary function $f(\xi)$ (see, e.g., Eq. (51) of Ref. [94] with $\lambda=0$ and Ref. [34]), 


$$
\begin{aligned}
x^{1}(\xi) & =x^{1}(0)+r^{1}(0) \xi-v_{-}^{-1} \int_{0}^{\xi} d x \int_{0}^{x} d y f(y) \cos \varphi(y), \\
x^{2}(\xi) & =x^{2}(0)+r^{2}(0) \xi-v_{-}^{-1} \int_{0}^{\xi} d x \int_{0}^{x} d y f(y) \sin \varphi(y), \\
x^{0}(\xi)+\zeta x^{3}(\xi) & =\int_{0}^{\xi} d x\left[v_{-}^{-2}+\left(r^{1}(0)-v_{-}^{-1} \int_{0}^{x} d y f(y) \cos \varphi(y)\right)^{2}+\left(r^{2}(0)-v_{-}^{-1} \int_{0}^{x} d y f(y) \sin \varphi(y)\right)^{2}\right], \\
\xi & =v_{-} \tau,
\end{aligned}
$$

where $r^{\mu}:=v^{\mu} / v_{-}, \quad v^{\mu}$ is the 4-velocity, $v_{\mu} v^{\mu}=1$, $v_{-}:=v^{0}-\zeta v^{3}=$ const, $\tau$ is the proper time, and it is assumed that $x^{0}=x^{3}=0$ at the initial instant of time. It is clear this assumption does not destroy the generality of our considerations. Upon shifting $x^{0}$ and $x^{3}$ by constants, the amplitude of radiation of a twisted photon changes by an overall phase, which does not affect the probability to record the twisted photon by detector. It is convenient to pass in formulas (32) and (38) from the integration variable $t$ to the variable $\xi$. The corresponding derivatives take the form

$$
\begin{aligned}
r^{1}(\xi) & =r^{1}(0)-v_{-}^{-1} \int_{0}^{\xi} d x f(x) \cos \varphi(x), \\
r^{2}(\xi) & =r^{2}(0)-v_{-}^{-1} \int_{0}^{\xi} d x f(x) \sin \varphi(x), \\
r^{0}(\xi)+\zeta r^{3}(\xi) & =v_{-}^{-2}+\left(r^{1}(0)-v_{-}^{-1} \int_{0}^{\xi} d x f(x) \cos \varphi(x)\right)^{2} \\
& +\left(r^{2}(0)-v_{-}^{-1} \int_{0}^{\xi} d x f(x) \sin \varphi(x)\right)^{2} .
\end{aligned}
$$

To obtain analytic formulas, we assume that the phase

$$
\varphi=\Omega \xi+\varphi_{0}
$$

where $\Omega$ is the frequency of the electromagnetic wave and $\varphi_{0}$ is the initial phase. The amplitude is chosen as

$$
f(\xi)=\text { const, } \quad \xi \in[0,2 \pi N],
$$

where $N$ is the number of periods of the electromagnetic wave. The amplitude vanishes outside this interval; i.e., it is assumed that the laser wave pulse possesses sharp rising and descending edges. In that case, employing the notation from Eqs. (32) and (38), we have

$$
\begin{aligned}
r_{ \pm} & =\bar{r}_{ \pm} \pm i K v_{-}^{-1} e^{ \pm i \varphi} \\
r^{0} & =\frac{1}{2 v_{-}^{2}}\left[v_{-}^{2}+1+K^{2}+\bar{v}_{\perp}^{2}-2 K \bar{v}_{\perp} \sin (\varphi-\rho)\right] \\
r^{3} & =\frac{\zeta}{2 v_{-}^{2}}\left[1+K^{2}+\bar{v}_{\perp}^{2}-v_{-}^{2}-2 K \bar{v}_{\perp} \sin (\varphi-\rho)\right]
\end{aligned}
$$

where $K:=f / \Omega$,

$$
\bar{r}_{ \pm}:=r_{ \pm}(0) \mp i K v_{-}^{-1} e^{ \pm i \varphi_{0}}, \quad \bar{v}_{\perp}:=v_{-}\left|\bar{r}_{ \pm}\right|,
$$

and $\rho=\arg \bar{r}_{+}$. The solution to the Lorentz equations is given by

$$
\begin{aligned}
x_{ \pm}= & \bar{x}_{ \pm}+\bar{r}_{ \pm} \xi+\frac{K}{v_{-} \Omega} e^{ \pm i \varphi} \\
x^{0}= & \frac{1}{2 v_{-}^{2}}\left[\left(v_{-}^{2}+1+K^{2}+\bar{v}_{\perp}^{2}\right) \xi+2 \bar{v}_{\perp} \frac{K}{\Omega}(\cos (\varphi-\rho)\right. \\
& \left.\left.-\cos \left(\varphi_{0}-\rho\right)\right)\right], \\
x^{3}= & \frac{\zeta}{2 v_{-}^{2}}\left[\left(1+K^{2}+\bar{v}_{\perp}^{2}-v_{-}^{2}\right) \xi+2 \bar{v}_{\perp} \frac{K}{\Omega}(\cos (\varphi-\rho)\right. \\
& \left.\left.-\cos \left(\varphi_{0}-\rho\right)\right)\right]
\end{aligned}
$$

where

$$
\bar{x}_{ \pm}:=x_{ \pm}(0)-\frac{K}{v_{-} \Omega} e^{ \pm i \varphi_{0}} .
$$

Notice that if the charged particle moves initially along the axis of the twisted photon detector, viz., $r_{ \pm}(0)=0$, then $\bar{r}_{ \pm} \neq 0$.

\section{A. Radiation without recoil}

Let us consider the radiation of twisted photons in the case in which the quantum recoil is negligible. To find the probability of radiation [(36), [33]], it is necessary to evaluate the amplitudes

$$
\begin{aligned}
I_{3} & =\int_{0}^{T N} d \xi r^{3}(\xi) e^{-i k_{0}\left(x^{0}-n_{3} x^{3}\right)} j_{m}\left(k_{\perp} x_{+}, k_{\perp} x_{-}\right) \\
I_{ \pm} & =\frac{i n_{\perp}}{s \mp n_{3}} \int_{0}^{T N} d \xi r_{ \pm}(\xi) e^{-i k_{0}\left(x^{0}-n_{3} x^{3}\right)} j_{m \mp 1}\left(k_{\perp} x_{+}, k_{\perp} x_{-}\right) .
\end{aligned}
$$

Then, the probability to record the twisted photon is 
$d P\left(s, m, k_{\perp}, k_{3}\right)=e^{2}\left|I_{3}+\frac{1}{2} I_{+}+\frac{1}{2} I_{-}\right|^{2} n_{\perp}^{3} \frac{d k_{3} d k_{\perp}}{16 \pi^{2}}$.

In formula (95), the contributions from the parts of the particle trajectory with $\xi \notin[0,2 \pi N]$ are neglected. These contributions correspond to the edge radiation. They can be ignored when the energies of recorded photons are sufficiently large. A thorough description of the edge radiation in terms of twisted photons is given in Ref. [100].

The evaluation of integrals (95) is performed analogously to the case of undulator radiation studied in Ref. [33]. First of all, we shift the integration variable $\xi \rightarrow \xi+T N / 2$ and make use of the addition theorem (A6) in Ref. [33] (see also Ref. [101]) for the Bessel functions,

$j_{m}\left(k_{\perp} x_{+}, k_{\perp} x_{-}\right)=\sum_{l=-\infty}^{\infty} j_{m-l}\left(k_{\perp} z_{+}, k_{\perp} z_{-}\right) j_{l}\left(k_{\perp} y_{+}, k_{\perp} y_{-}\right)$

where

$$
z_{ \pm}:=\bar{x}_{ \pm}+\bar{r}_{ \pm} T N / 2, \quad y_{ \pm}:=\bar{r}_{ \pm} \xi+\frac{K}{v_{-} \Omega} e^{ \pm i \varphi} .
$$

Now, the phase $\varphi_{0}$ entering into $\varphi$ includes $\pi N$. We denote this phase by $\varphi_{0 N}$. Notice that, on shifting the variable $\xi$, the phase $\varphi_{0}$ appearing explicitly in formulas (92), (93), and (94) does not change. Substitute the integral representation

$j_{l}\left(k_{\perp} y_{+}, k_{\perp} y_{-}\right)=i^{-l} \int_{-\pi}^{\pi} \frac{d \psi}{2 \pi} e^{i l \psi} e^{i k_{\perp}\left(y_{2} \sin \psi+y_{1} \cos \psi\right)}$

into (97) and then (97) into (95). As a result, the expression standing in the exponent in the integrand of $I_{3}$ becomes

$$
\begin{array}{r}
-i \frac{k_{0}}{2 v_{-}^{2}} \xi\left[\left(1-\zeta n_{3}\right)\left(1+K^{2}+\bar{v}_{\perp}^{2}\right)+\left(1+\zeta n_{3}\right) v_{-}^{2}\right. \\
\left.-2 n_{\perp} \bar{v}_{\perp} v_{-} \cos (\psi-\rho)\right]+i \eta \sin (\varphi+\delta)+i l \psi
\end{array}
$$

up to a constant term that does not influence the probability of radiation. Here,

$$
\begin{aligned}
\eta \cos \delta & :=\frac{K k_{0}}{v_{-} \Omega}\left(n_{\perp} \sin \psi-\left(1-\zeta n_{3}\right) \bar{r}^{2}\right), \\
\eta \sin \delta & :=\frac{K k_{0}}{v_{-} \Omega}\left(n_{\perp} \cos \psi-\left(1-\zeta n_{3}\right) \bar{r}^{1}\right) .
\end{aligned}
$$

Using the Fourier series expansion

$$
e^{i \eta \sin (\varphi+\delta)}=\sum_{n=-\infty}^{\infty} e^{i n(\varphi+\delta)} J_{n}(\eta)
$$

the integral over $\xi$ is reduced to the deltalike sequence
$\int_{-T N / 2}^{T N / 2} \frac{d \xi}{2 \pi} e^{-i x_{n} \xi}=\delta_{N}(x), \quad \delta_{N}\left(x_{n}\right):=\frac{\sin \left(T N x_{n} / 2\right)}{\pi x_{n}}$.

The argument of the regularized delta function reads

$$
\begin{aligned}
x_{n}= & \frac{k_{0}}{2 v_{-}^{2}}\left[\left(1-\zeta n_{3}\right)\left(1+K^{2}+\bar{v}_{\perp}^{2}\right)+\left(1+\zeta n_{3}\right) v_{-}^{2}\right. \\
& \left.-2 n_{\perp} \bar{v}_{\perp} v_{-} \cos (\psi-\rho)\right]-\Omega n .
\end{aligned}
$$

For $N$ large, the main contribution to the integral over $\psi$ comes from the points where the argument of the regularized delta function vanishes.

Below, we shall assume that $\Omega>0$ and, at the end, shall discuss how the results change for $\Omega<0$. The condition $x_{n}=0$ can be conveniently written as

$$
x_{n}=\frac{\Omega}{2}\left(b_{n}+a_{n}\right)\left[\cos \xi_{n}-\cos (\psi-\rho)\right]=0,
$$

where the notation has been introduced [33]

$$
\begin{aligned}
& a_{n}:=n-k_{0} \omega_{+}^{-1}, \quad b_{n}:=k_{0} \omega_{-}^{-1}-n, \\
& \xi_{n}:=\arccos \frac{b_{n}-a_{n}}{b_{n}+a_{n}},
\end{aligned}
$$

and

$\omega_{ \pm}:=\frac{2 \Omega v_{-}^{2}}{\left(1-\zeta n_{3}\right)\left(1+K^{2}+\bar{v}_{\perp}^{2}\right)+\left(1+\zeta n_{3}\right) v_{-}^{2} \mp 2 n_{\perp} \bar{v}_{\perp} v_{-}}$

If $N$ is so large that $\delta_{N}\left(x_{n}\right)$ removes the integration over $\psi$, then the energy spectrum of radiated twisted photons consists of the intervals

$$
k_{0} \in n\left[\omega_{-}, \omega_{+}\right], \quad n=\overline{1, \infty}
$$

The radiation is suppressed outside these intervals. These intervals become overlapping starting from the harmonic number

$$
n_{0}=\frac{\omega_{-}}{\omega_{+}-\omega_{-}} .
$$

When $k_{0}$ belongs to the intervals, $a_{n} \geq 0, b_{n} \geq 0$, and $\xi_{n} \in[0, \pi]$.

As a result, neglecting the terms at nonpositive $n$, we have

$$
\begin{aligned}
I_{3} \approx & \sum_{n=1}^{\infty} \sum_{l=-\infty}^{\infty} j_{m-l}\left(k_{\perp} z_{+}, k_{\perp} z_{-}\right) \int_{-\pi}^{\pi} d \psi \delta_{N}\left(x_{n}\right) e^{i n\left(\delta+\varphi_{0 N}\right)+i l \psi} i^{-l} \\
& \times \frac{\zeta}{2 v_{-}^{2}}\left[\left(1+K^{2}+\bar{v}_{\perp}^{2}-v_{-}^{2}\right) J_{n}(\eta)\right. \\
& \left.+i K \bar{v}_{\perp}\left(e^{-i(\delta+\rho)} J_{n-1}(\eta)-e^{i(\delta+\rho)} J_{n+1}(\eta)\right)\right]
\end{aligned}
$$


As for the rest integrals, we obtain similarly

$$
I_{ \pm} \approx \sum_{n=1}^{\infty} \sum_{l=-\infty}^{\infty} j_{m-l}\left(k_{\perp} z_{+}, k_{\perp} z_{-}\right) \int_{-\pi}^{\pi} d \psi \delta_{N}\left(x_{n}\right) e^{i n\left(\delta+\varphi_{0 N}\right)+i(l \mp 1) \psi} i^{-l} \frac{\mp n_{\perp}}{s \mp n_{3}}\left[\bar{r}_{ \pm} J_{n} \pm i K v_{-}^{-1} J_{n \mp 1} e^{\mp i \delta}\right],
$$

where the arguments of the Bessel functions are the same as in (110). To obtain (111), one needs to shift the summation index $l \rightarrow l \mp 1$ in the series (97). Taking into account estimates (C7), the total contribution to the radiation amplitude takes the form

$$
I_{3}+\frac{1}{2}\left(I_{+}+I_{-}\right) \approx \sum_{n=1}^{\infty} \sum_{l=-\infty}^{\infty} j_{m-l}\left(k_{\perp} z_{+}, k_{\perp} z_{-}\right) \int_{-\pi}^{\pi} \frac{d \psi}{2} \delta_{N}\left(x_{n}\right) e^{i n\left(\delta+\varphi_{0 N}\right)+i l \psi} i^{-l} g_{n}(\psi),
$$

where

$$
g_{n}(\psi):=\zeta \frac{1+K^{2}+\bar{v}_{\perp}^{2}-v_{-}^{2}}{v_{-}^{2}} J_{n}+\zeta \frac{i K \bar{v}_{\perp}}{v_{-}^{2}}\left(e^{-i(\delta+\rho)} J_{n-1}-e^{i(\delta+\rho)} J_{n+1}\right)-\frac{2}{n_{\perp}} e^{-i s \psi}\left(\bar{r}_{s} J_{n}+s \frac{i K}{v_{-}} J_{n-s} e^{-i s \delta}\right) .
$$

The last expression can be rewritten in terms of the Bessel function and its derivative with the same index:

$$
\begin{aligned}
g_{n}(\psi)= & 2\left[\zeta \frac{1+K^{2}+\bar{v}_{\perp}^{2}-v_{-}^{2}}{2 v_{-}^{2}}-\frac{i s K n}{n_{\perp} v_{-} \eta} e^{-i s(\delta+\psi)}+\zeta \frac{K \bar{v}_{\perp} n}{v_{-}^{2} \eta} \sin (\delta+\rho)-\frac{\bar{r}_{s}}{n_{\perp}} e^{-i s \psi}\right] J_{n} \\
& -\frac{2 i K}{n_{\perp} v_{-}}\left[e^{-i s(\delta+\psi)}-\zeta \frac{n_{\perp} \bar{v}_{\perp}}{v_{-}} \cos (\delta+\rho)\right] J_{n}^{\prime} .
\end{aligned}
$$

Further, we suppose that $N$ is so large that $\delta_{N}\left(x_{n}\right)$ removes the integration over $\psi$. The solution of (105) is, evidently,

$$
\psi=\rho \pm \xi_{n} .
$$

As in the case of undulator radiation, the three cases occur [33]: (a) the regular case $\xi_{n} \neq\{0, \pi\}$, (b) the weakly degenerate case $\xi_{n}=\{0, \pi\}$, and (c) the strongly degenerate case $a_{n}=b_{n}=0$.

Let us begin with the regular case. For $N$ large, in the leading order, we deduce
$\delta_{N}\left(x_{n}\right) \approx \frac{\theta\left(a_{n}\right) \theta\left(b_{n}\right)}{\Omega \sqrt{a_{n} b_{n}}}\left[\delta\left(\psi-\rho-\xi_{n}\right)+\delta\left(\psi-\rho+\xi_{n}\right)\right]$.

The delta functions remove integration in (112). The remaining sum over $l$ can be performed by using the relation

$$
\sum_{k=-\infty}^{\infty} t^{k} j_{k}(p, q)=e^{(p t-q / t) / 2}
$$

which follows from Eq. (A7) in Ref. [33]. Then, up to an irrelevant phase,

$I_{3}+\frac{1}{2}\left(I_{+}+I_{-}\right) \approx \sum_{n=1}^{\infty} \frac{\theta\left(a_{n}\right) \theta\left(b_{n}\right)}{2 \Omega \sqrt{a_{n} b_{n}}} e^{i n \varphi_{0 N}}\left\{\left.e^{i n \delta+i m \psi+i k_{\perp}\left|z_{+}\right| \cos \left(\psi-\arg z_{+}\right)} g_{n}(\psi)\right|_{\psi=\rho+\xi_{n}}+\left.e^{i n \delta+i m \psi+i k_{\perp}\left|z_{+}\right| \cos \left(\psi-\arg z_{+}\right)} g_{n}(\psi)\right|_{\psi=\rho-\xi_{n}}\right\}$

In the photon energy range in which the harmonics do not overlap, we obtain

$$
\begin{aligned}
d P\left(s, m, k_{\perp}, k_{3}\right) \approx & e^{2} \sum_{n=1}^{\infty} \frac{\theta\left(a_{n}\right) \theta\left(b_{n}\right)}{4 \Omega^{2} a_{n} b_{n}}\left|e^{i n \delta+i m \psi+i k_{\perp}\left|z_{+}\right| \cos \left(\psi-\arg z_{+}\right)} g_{n}(\psi)\right|_{\psi=\rho+\xi_{n}} \\
& +\left.\left.e^{i n \delta+i m \psi+i k_{\perp}\left|z_{+}\right| \cos \left(\psi-\arg z_{+}\right)} g_{n}(\psi)\right|_{\psi=\rho-\xi_{n}}\right|^{2} n_{\perp}^{3} \frac{d k_{3} d k_{\perp}}{16 \pi^{2}} .
\end{aligned}
$$

The dependence on $m$ for the energies $k_{0}$ belonging to the spectral band with number $n$ is periodic with the period [33] 


$$
T_{m}= \begin{cases}\pi / \xi_{n}, & \xi_{n} \in(0, \pi / 2) \\ \pi /\left(\pi-\xi_{n}\right), & \xi_{n} \in[\pi / 2, \pi)\end{cases}
$$

Of course, this periodicity holds only for those quantum numbers $m$ where $\delta_{N}\left(x_{n}\right)$ can be replaced by delta function (116).

Now, we turn to the weakly degenerate case. Let $a_{n}=0$, $b_{n}>0$, i.e., $k_{0}=n \omega_{+}$. Then,

$$
b_{n}=n\left(\omega_{+} \omega_{-}^{-1}-1\right), \quad \xi_{n}=0, \quad \psi=\rho .
$$

We assume that $N$ is so large that all the integrand functions in (112), apart form $\delta_{N}\left(x_{n}\right)$, can be taken at the point $\psi=\rho$ and be removed from the integrand. In that case, the integral arises:

$$
\begin{aligned}
\int_{-\pi}^{\pi} d \psi \delta_{N}\left(x_{n}\right) & \approx \int_{-\infty}^{\infty} d \psi \frac{\sin \left[\pi N n\left(\omega_{+} \omega_{-}^{-1}-1\right) \psi^{2} / 4\right]}{\pi \Omega n\left(\omega_{+} \omega_{-}^{-1}-1\right) \psi^{2} / 4} \\
& =\Omega^{-1} \sqrt{\frac{8 N}{n\left(\omega_{+} \omega_{-}^{-1}-1\right)}} .
\end{aligned}
$$

The probability to record a twisted photon becomes

$d P\left(s, m, k_{\perp}, k_{3}\right) \approx e^{2} \frac{N\left|g_{n}(\rho)\right|^{2} n_{\perp}^{3}}{\Omega^{2} n\left(\omega_{+} \omega_{-}^{-1}-1\right)} \frac{d k_{3} d k_{\perp}}{8 \pi^{2}}$.

For $b_{n}=0, a_{n}>0$, i.e., for $k_{0}=n \omega_{-}$, the similar calculations lead to

$$
d P\left(s, m, k_{\perp}, k_{3}\right) \approx e^{2} \frac{N\left|g_{n}(\pi-\rho)\right|^{2} n_{\perp}^{3}}{\Omega^{2} n\left(1-\omega_{-} \omega_{+}^{-1}\right)} \frac{d k_{3} d k_{\perp}}{8 \pi^{2}} .
$$

In the domain where the applicability conditions of the approximations made are fulfilled, the explicit dependence of the twisted photon radiation probability on $m$ disappears.

In the strongly degenerate case, the spectral bands (108) turn into narrow lines $k_{0}=n \omega_{+}=n \omega_{-}$. This happens when $\bar{v}_{\perp} \approx 0$. Then,

$$
\eta=\frac{K k_{\perp}}{v_{-} \Omega}, \quad \delta=\pi / 2-\psi .
$$

The functions,

$$
\begin{aligned}
x_{n} & =\frac{k_{0}}{2 v_{-}^{2}}\left[\left(1-\zeta n_{3}\right)\left(1+K^{2}\right)+\left(1+\zeta n_{3}\right) v_{-}^{2}\right]-\Omega n, \\
g_{n} & =\zeta \frac{1+K^{2}-v_{-}^{2}}{v_{-}^{2}} J_{n}-\frac{2 K}{n_{\perp} v_{-}} J_{n-s} \\
& =\left[\zeta \frac{1+K^{2}-v_{-}^{2}}{v_{-}^{2}}-\frac{2 \Omega n}{n_{\perp} k_{\perp}}\right] J_{n}-\frac{2 s K}{n_{\perp} v_{-}} J_{n}^{\prime},
\end{aligned}
$$

do not depend on $\psi$, and the integral over $\psi$ in (112) is readily performed. As a result,
$I_{3}+\frac{1}{2}\left(I_{+}+I_{-}\right) \approx \pi \delta_{N}\left(x_{n}\right) j_{m-n}\left(k_{\perp} z_{+}, k_{\perp} z_{-}\right) e^{i n \varphi_{0}}(-1)^{n N} g_{n}$.

The probability to record a twisted photon is given by

$d P\left(s, m, k_{\perp}, k_{3}\right)=e^{2} \delta_{N}^{2}\left(x_{n}\right) J_{m-n}^{2}\left(k_{\perp}\left|z_{+}\right|\right) g_{n}^{2} n_{\perp}^{3} \frac{d k_{3} d k_{\perp}}{16}$.

If $k_{\perp}\left|z_{+}\right| \ll 1$, then the selection rule $m=n$ is fulfilled $[33,42,46,50-56]$.

Let us show how the above results are modified for $\Omega<0$. The sign change of $\Omega$ corresponds to a change of polarization of the incident electromagnetic wave (85). This, in turn, leads to a change of handedness of the helix along which the charged particle is moving. Upon changing the sign of $\Omega$, formulas (106)-(109) remain valid with the replacement $\Omega \rightarrow|\Omega|$. Since the substitution $\Omega \rightarrow-\Omega$ results in

$$
K \rightarrow-K, \quad n \rightarrow-n,
$$

we have

$$
g_{n}(\psi) \rightarrow(-1)^{n} g_{n}(\psi)
$$

Therefore, on substituting $\Omega \rightarrow-|\Omega|$ in (119), (123), (124), and (128), one must set

$$
e^{i n \delta} \rightarrow e^{-i n \delta}
$$

in formula (119); formulas (123) and (124) remain intact; and in formula (128), one needs to replace

$$
J_{m-n}^{2}\left(k_{\perp}\left|z_{+}\right|\right) \rightarrow J_{m+n}^{2}\left(k_{\perp}\left|z_{+}\right|\right)
$$

For $k_{\perp}\left|z_{+}\right| \ll 1$, the selection rule in the strongly degenerate case looks like $m=-n$.

Consider in more detail the cases in which the electromagnetic wave propagates toward the detector of twisted photons or from it. In these cases, the electron bunch moves approximately along the direction of propagation of the electromagnetic wave or in the opposite direction, respectively. In the case in which the electromagnetic wave propagates toward the detector, we have $\zeta=1$ and

$v_{-} \approx \frac{1+v_{\perp}^{2}}{2 \gamma} \sim \frac{\varkappa^{2}}{2 \gamma}, \quad v_{\perp} \sim \varkappa, \quad \bar{v}_{\perp} \lesssim \varkappa, \quad n_{\perp} \lesssim \frac{\varkappa}{\gamma}$.

The bounds of the spectral bands (108) are expressed through

$\omega_{ \pm} \approx \Omega\left[\left(1 \mp \frac{n_{\perp} \bar{v}_{\perp}}{2 v_{-}}\right)^{2}+\frac{n_{\perp}^{2}}{4 v_{-}^{2}}\left(1+K^{2}\right)\right]^{-1} \sim \Omega$. 
In the strongly degenerate case, the radiation spectrum becomes

$$
k_{0} \approx \Omega n\left[1+\frac{n_{\perp}^{2}}{4 v_{-}^{2}}\left(1+K^{2}\right)\right]^{-1}, \quad n=\overline{1, \infty} .
$$

For $n \geq 5$, the Bessel functions entering into $g_{n}$ can be expressed through the Airy functions (see Eq. (122) in Ref. [33] and also Ref. [9]) with

$$
x=1-\frac{16 K^{2} v_{-}^{2} / n_{\perp}^{4}}{\left(1+K^{2}+4 v_{-}^{2} / n_{\perp}^{2}\right)^{2}} .
$$

For the radiation probability not to be exponentially suppressed, this quantity should be small $x \lesssim 1 / 20$. This occurs for $K \gtrsim 3$ and

$$
n_{\perp} \approx \frac{2 v_{-}}{K} \approx \frac{1+v_{\perp}^{2}}{\gamma K} \sim \frac{\varkappa}{\gamma}
$$

In that case, the radiation probability drops exponentially to zero at the harmonic numbers [33]

$$
n \gtrsim \frac{K^{3}}{\sqrt{2}} .
$$

If the electromagnetic wave moves from the detector, i.e., the head-on collision of the laser wave with the bunch of charged particles is considered, then $\zeta=-1$, and

$$
v_{-} \approx 2 \gamma, \quad \bar{v}_{\perp} \lesssim \varkappa, \quad n_{\perp} \lesssim \varkappa / \gamma,
$$

and also

$$
\omega_{ \pm} \approx \frac{\Omega v_{-}^{2}}{1+K^{2}+\left(\bar{v}_{\perp} \mp n_{\perp} v_{-} / 2\right)^{2}} \sim \frac{4 \Omega \gamma^{2}}{\varkappa^{2}} .
$$

The analysis in this case is completely analogous to the analysis of the undulator radiation [33] at the observation angle $\theta:=\bar{v}_{\perp} / \gamma$ and the undulator frequency $\omega:=2 \Omega$ (cf. Eq. (85) of Ref. [33]). In the strongly degenerate case, the radiation spectrum looks like

$$
k_{0} \approx \frac{\Omega n v_{-}^{2}}{1+K^{2}+n_{\perp}^{2} v_{-}^{2} / 4}, \quad n=\overline{1, \infty} .
$$

For $n \geq 5$, the radiation probability is not exponentially suppressed at $K \gtrsim 3$ if $n_{\perp} \approx K / \gamma$ and $n \lesssim K^{3} / \sqrt{2}$.

\section{B. Radiation with recoil}

Now, we take the quantum recoil into account. In the case $\zeta=1$, estimate (134) holds. Therefore, the quantum recoil can be neglected for reasonable photon energies of the laser wave [see Eq. (86)]. In that case, the probability to record a twisted photon radiated by both scalar and Dirac particles is described by formula (36) in Ref. [33]. Thus, formulas obtained above remain intact with good accuracy.

In the case $\zeta=-1$, the quantum recoil can be significant. Since the approximate equality (139) is valid, the quantity

$$
\begin{aligned}
q & =P_{0} / P_{0}^{\prime}=v_{0} / v_{0}^{\prime}=\gamma /\left(\gamma-k_{0}\right) \\
& \approx v_{-} /\left(v_{-}-2 k_{0}\right)=\mathrm{const},
\end{aligned}
$$

up to the terms of order $\varkappa^{2} / \gamma^{2}$. Hence, the probability to detect a twisted photon radiated by a charged scalar particle with the quantum recoil taken into account (32) is obtained from the formulas above, where the recoil was ignored, by multiplying the probability by $q$ and substituting

$$
k_{0} \rightarrow \bar{k}_{0}:=q k_{0}
$$

in the definitions of $a_{n}, b_{n}$ (106) and the radiation spectrum (108). As was discussed in the preceding sections, the term standing in the exponent and proportional to $k_{\perp}^{2} /\left(2 P_{0 i}\right)$ can be safely neglected. As far as the strongly degenerate case (128) is concerned, substitution (143) has to be done in formula (126) for $x_{n}$, and, of course, (128) must be multiplied by $q$.

The treatment of the Dirac particle case is a bit more complex. The probability to record a twisted photon equals

$d P\left(s, m, k_{3}, k_{\perp}\right)=d P_{1}\left(s, m, k_{\perp}, k_{3}\right)+d P_{a}\left(s, m, k_{\perp}, k_{3}\right)$,

where $d P_{1}\left(s, m, k_{\perp}, k_{3}\right)$ is the contribution of the first term in (38) and $d P_{a}\left(s, m, k_{\perp}, k_{3}\right)$ is the contribution of the second and third terms in (38). As long as (142) holds, the contribution of the first term in (38) is evaluated as in the case of negligible quantum recoil: the probability to record a twisted photon without recoil must be multiplied by

$$
(1+q)^{2} / 4,
$$

and substitution (143) must be performed in the definitions of $a_{n}$ and $b_{n}$ (106) and the radiation spectrum (108).

The contribution of the last two terms in (38) has to be evaluated from scratch. It follows from the explicit expressions for the mode functions [see Eq. (13) in Ref. [33]) that for $s=-1$ the third term in (38) can be omitted, while for $s=1$, the second term can be thrown out. Let

$I_{a}:=\frac{k_{0}}{4 v_{0}^{\prime}} \int_{0}^{T N} d \xi e^{-i \bar{k}_{0}\left(x^{0}-n_{3} x^{3}\right)}\left[r_{s} a_{-s}-i s n_{\perp} r^{0} a_{-s}(m+s)\right]$.

The contribution of the last two terms in (38) is proportional to the modulus squared of this integral. For the headon collision, we have

$$
r^{0} \approx 1 / 2
$$


Then, performing the calculations completely analogous to the case of negligible recoil, we find

$$
\begin{aligned}
I_{a} \approx & \frac{k_{0}}{4 v_{0}^{\prime}} \sum_{n=1}^{\infty} \sum_{l=-\infty}^{\infty} j_{m-l}\left(k_{\perp} z_{+}, k_{\perp} z_{-}\right) \\
& \times \int_{-\pi}^{\pi} d \psi \delta_{N}\left(x_{n}\right) e^{i n\left(\delta+\varphi_{0 N}\right)+i l \psi} i^{-l} g_{n}^{a}(\psi),
\end{aligned}
$$

where

$g_{n}^{a}(\psi):=\left(1-2 \frac{\bar{r}_{s}}{n_{\perp}} e^{-i s \psi}\right) J_{n}(\eta)-\frac{2 i s K}{n_{\perp} v_{-}} J_{n-s}(\eta) e^{-i s(\delta+\psi)}$, and $x_{n}$ has the form (105) with

$$
\begin{aligned}
& a_{n}:=n-\bar{k}_{0} \omega_{+}^{-1}, \quad b_{n}:=\bar{k}_{0} \omega_{-}^{-1}-n, \\
& \xi_{n}:=\arccos \frac{b_{n}-a_{n}}{b_{n}+a_{n}} .
\end{aligned}
$$

The approximate expressions for $\omega_{ \pm}$are written in (140).

Let us consider separately the regular, weakly degenerate, and strongly degenerate cases. In the regular case, under the same assumptions that were made in considering the first term in (38), we deduce up to an irrelevant phase,

$I_{a} \approx \frac{k_{0}}{4 v_{0}^{\prime}} \sum_{n=1}^{\infty} \frac{\theta\left(a_{n}\right) \theta\left(b_{n}\right)}{\Omega \sqrt{a_{n} b_{n}}} e^{i n \varphi_{0 N}}\left\{\left.e^{i n \delta+i m \psi+i k_{\perp}\left|z_{+}\right| \cos \left(\psi-\arg z_{+}\right)} g_{n}^{a}(\psi)\right|_{\psi=\rho+\xi_{n}}+\left.e^{i n \delta+i m \psi+i k_{\perp}\left|z_{+}\right| \cos \left(\psi-\arg z_{+}\right)} g_{n}^{a}(\psi)\right|_{\psi=\rho-\xi_{n}}\right\}$.

The respective contribution to the probability, in the region of photon energies where the harmonics do not overlap, becomes

$$
\begin{aligned}
d P_{a}\left(s, m, k_{\perp}, k_{3}\right) \approx & \frac{e^{2} k_{0}^{2}}{16 v^{\prime 2}} \sum_{n=1}^{\infty} \frac{\theta\left(a_{n}\right) \theta\left(b_{n}\right)}{\Omega^{2} a_{n} b_{n}}\left|e^{i n \delta+i m \psi+i k_{\perp}\left|z_{+}\right| \cos \left(\psi-\arg z_{+}\right)} g_{n}^{a}(\psi)\right|_{\psi=\rho+\xi_{n}} \\
& +\left.\left.e^{i n \delta+i m \psi+i k_{\perp}\left|z_{+}\right| \cos \left(\psi-\arg z_{+}\right)} g_{n}^{a}(\psi)\right|_{\psi=\rho-\xi_{n}}\right|^{2} n_{\perp}^{3} \frac{d k_{3} d k_{\perp}}{16 \pi^{2}}
\end{aligned}
$$

This expression, just as the contribution of the first term in (38), is a periodic function of $m$ with the period given in (120).

In the weakly degenerate case, for $a_{n}=0, b_{n}>0$, i.e., $\bar{k}_{0}=n \omega_{+}$, we obtain

$d P_{a}\left(s, m, k_{\perp}, k_{3}\right) \approx e^{2} \frac{k_{0}^{2}}{v_{0}^{\prime 2}} \frac{N\left|g_{n}^{a}(\rho)\right|^{2} n_{\perp}^{3}}{\Omega^{2} n\left(\omega_{+} \omega_{-}^{-1}-1\right)} \frac{d k_{3} d k_{\perp}}{32 \pi^{2}}$.

If $b_{n}=0, a_{n}>0$, i.e., $\bar{k}_{0}=n \omega_{-}$, then

$d P_{a}\left(s, m, k_{\perp}, k_{3}\right) \approx e^{2} \frac{k_{0}^{2}}{v_{0}^{\prime 2}} \frac{N\left|g_{n}^{a}(\pi-\rho)\right|^{2} n_{\perp}^{3}}{\Omega^{2} n\left(1-\omega_{-} \omega_{+}^{-1}\right)} \frac{d k_{3} d k_{\perp}}{32 \pi^{2}}$.

These expressions are independent of $m$.

In the strongly degenerate case $a_{n}=0, b_{n}=0$, we suppose that $\bar{v}_{\perp}=0$. Then, $x_{n}$ does not depend on $\psi$,

$$
\begin{aligned}
g_{n}^{a} & =J_{n}(\eta)-\frac{2 K}{n_{\perp} v_{-}} J_{n-s}(\eta) \\
& =\left(1-\frac{2 n \Omega}{n_{\perp} k_{\perp}}\right) J_{n}(\eta)-\frac{2 s K}{n_{\perp} v_{-}} J_{n}^{\prime}(\eta)=g_{n},
\end{aligned}
$$

and relations (125) take place. The contribution to the probability to detect a twisted photon is

$$
\begin{aligned}
& d P_{a}\left(s, m, k_{\perp}, k_{3}\right) \\
& \quad=e^{2} \frac{k_{0}^{2}}{v_{0}^{\prime 2}} \delta_{N}^{2}\left(x_{n}\right) J_{m-n}^{2}\left(k_{\perp}\left|z_{+}\right|\right) g_{n}^{2} n_{\perp}^{3} \frac{d k_{3} d k_{\perp}}{64} .
\end{aligned}
$$

The total probability (144) becomes [cf. Eq. (54)]

$$
\begin{aligned}
& d P\left(s, m, k_{\perp}, k_{3}\right) \\
& \quad=e^{2}\left(1+q^{2}\right) \delta_{N}^{2}\left(x_{n}\right) J_{m-n}^{2}\left(k_{\perp}\left|z_{+}\right|\right) g_{n}^{2} n_{\perp}^{3} \frac{d k_{3} d k_{\perp}}{32} .
\end{aligned}
$$

For $k_{\perp}\left|z_{+}\right| \ll 1$, the selection rule $m=n$ is fulfilled. As in the case of forward undulator radiation, the applicability conditions (74) and (84) must be satisfied. The number of twisted photons recorded by the detector is approximately given by (73).

\section{Examples}

As is seen from (128) and (157), the electrons moving in the laser wave represent a pure source of twisted photons only in the strongly degenerate case in which $\bar{v}_{\perp} \approx 0$ and $k_{\perp}\left|z_{+}\right| \approx 0$. For this to be the case, the initial data must be taken in the form [see (92), (94), and (98)]

$$
r_{+}(0)=i K v_{-}^{-1} e^{i \varphi_{0}}, \quad x_{+}(0)=K v_{-}^{-1} \Omega^{-1} e^{i \varphi_{0}} .
$$

Since it is very hard to control the initial phase of a laser wave, in the wiggler regime, $K \gtrsim 3$, it is practically 

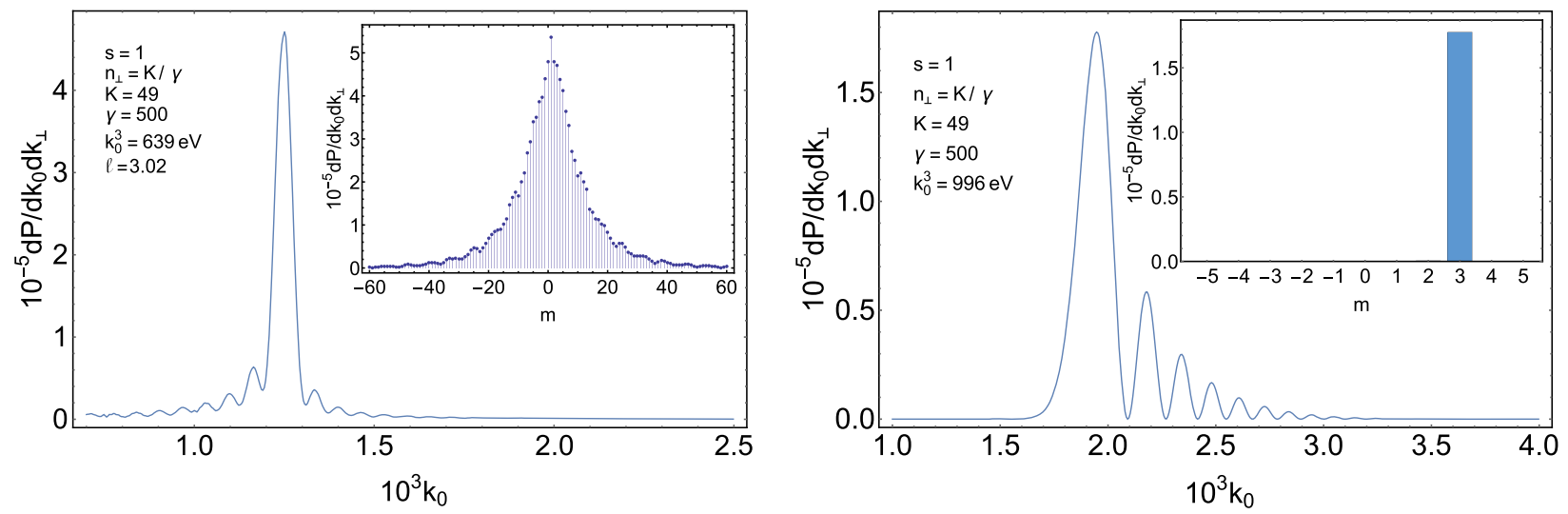

FIG. 3. The radiation of twisted photons in head-on collision of $256 \mathrm{MeV}$ electrons with the circularly polarized electromagnetic wave produced by the Ti:Sa laser. The parameters are the same as in Fig. 6, but the laser pulse amplitude envelopes are different. Left panel: The amplitude envelope has the form (90). The initial laser wave phase $\varphi_{0}=0$. The maximum near $k_{0}=3 \omega_{+}$is shown. Right panel: The amplitude envelope is $f_{0} \sin ^{4}(\Omega \xi /(2 N))$. The third harmonic (166) is depicted. Insets: The distribution over $m$ at the main maximum.

impossible to launch the electron to the electromagnetic wave so that the radiation produced by it would correspond to the strongly degenerate case. For an arbitrarily chosen phase, equalities (158) are strongly violated, harmonics
(108) spread violently, and the twisted photon detector records a wide distribution over $m$ (see Fig. 3). In this case, the twisted photons escape the laser wave at large angles to the detector axis rather than move along it (see Fig. 4).
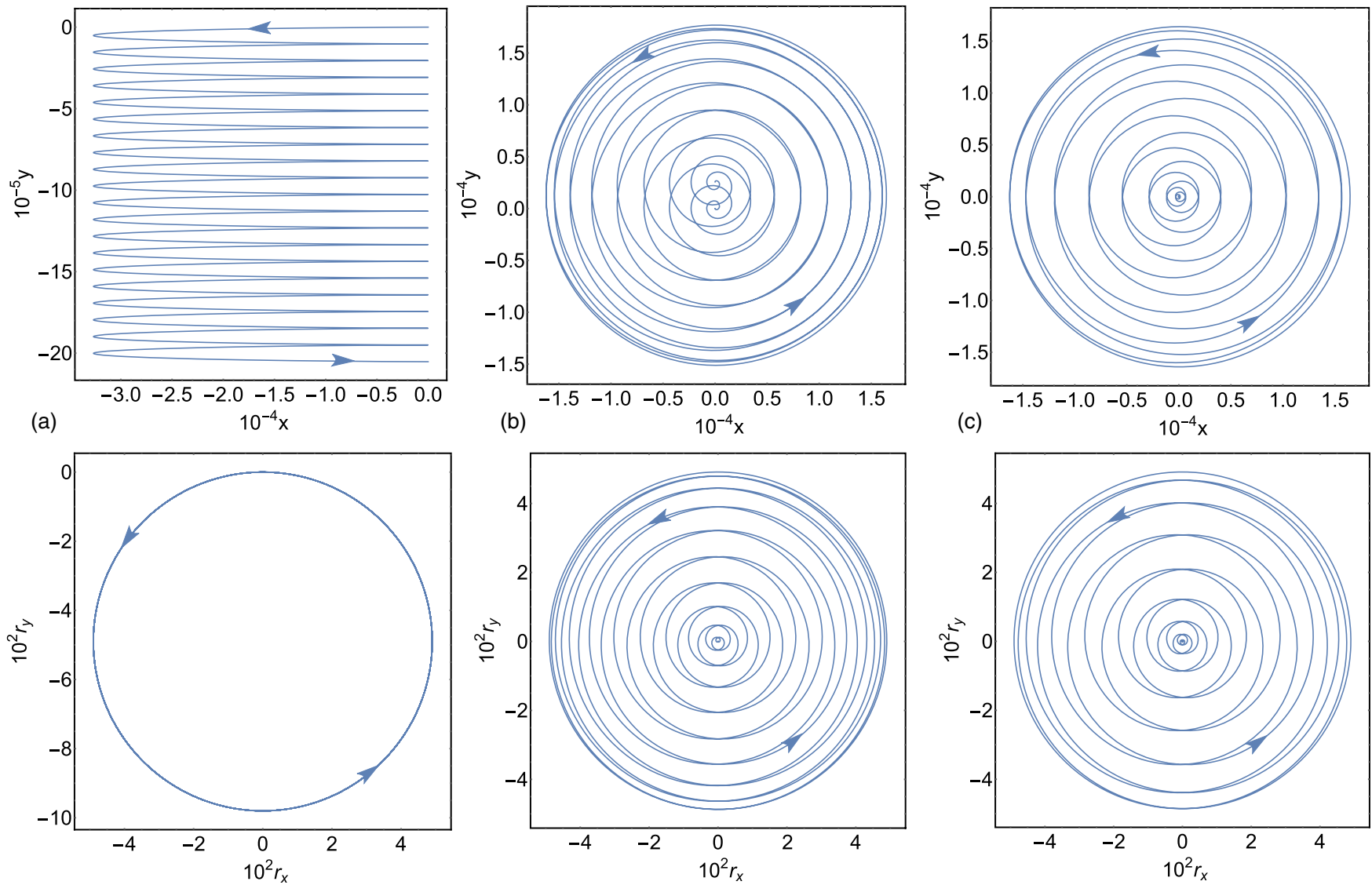

FIG. 4. The coordinates $x, y$ and "velocities" $r_{x}, r_{y}$ for head-on collision of an electron with the circularly polarized electromagnetic wave produced by the Ti:Sa laser. The parameters are the same as in Fig. 3. The electron moves initially along the detector axis. The initial laser wave phase $\varphi_{0}=0$. The lengths are measured in the Compton wavelengths. a) The amplitude envelope has the form (90). b) The amplitude envelope is given in (163). c) The amplitude envelope is $f_{0} \sin ^{4}(\Omega \xi /(2 N))$. 
It turns out that this situation can be improved if one takes into account that, usually, the laser wave pulses generated in experiments have no sharp rising and descending edges. The amplitude envelope $f(\xi)$ is a smooth function vanishing at $\xi=\{0, T N\}$ and $f^{\prime}(\xi) / f(\xi) \sim 1 / N$. For $N \gtrsim 10$, this entails that the radiation probability ceases to depend virtually on the initial phase $\varphi_{0}$. As a result, it is possible to choose the initial velocity and entrance point of the electron to the electromagnetic wave so that the corresponding radiation will be a sufficiently pure source of twisted photons.

Indeed, under the restrictions on the form of the envelope mentioned above, the integrals entering into the general solution (87), (88) of the Lorentz equations can be approximately evaluated for $N \gtrsim 10$. Integrating once by parts and keeping only the integrated term, we obtain [cf. Eq. (91) and (93)]

$$
\begin{aligned}
x_{ \pm} & \approx x_{ \pm}(0)+r_{ \pm}(0) \xi+\frac{K}{v_{-} \Omega} e^{ \pm i \varphi}, \\
x^{0} & \approx \frac{1}{2 v_{-}^{2}}\left[\left(v_{-}^{2}+1+K^{2}+v_{\perp}^{2}(0)\right) \xi+2 v_{\perp}(0) \frac{K}{\Omega} \cos (\varphi-\rho)\right], \\
x^{3} & \approx \frac{\zeta}{2 v_{-}^{2}}\left[\left(1+K^{2}+v_{\perp}^{2}(0)-v_{-}^{2}\right) \xi+2 v_{\perp}(0) \frac{K}{\Omega} \cos (\varphi-\rho)\right],
\end{aligned}
$$

where $K:=f(\xi) / \Omega$ and

$$
\begin{aligned}
& r_{ \pm} \approx r_{ \pm}(0) \pm i K v_{-}^{-1} e^{ \pm i \varphi}, \\
& r^{0} \approx \frac{1}{2 v_{-}^{2}}\left[v_{-}^{2}+1+K^{2}+v_{\perp}^{2}(0)-2 K v_{\perp}(0) \sin (\varphi-\rho)\right], \\
& r^{3} \approx \frac{\zeta}{2 v_{-}^{2}}\left[1+K^{2}+v_{\perp}^{2}(0)-v_{-}^{2}-2 K v_{\perp}(0) \sin (\varphi-\rho)\right] .
\end{aligned}
$$

The accuracy of this approximation increases as $N$ increases. As is seen, the form of the trajectory is almost the same as in the case of a laser wave with constant amplitude but without strong dependence on the initial phase. Now, the dependence on the initial phase is contained only in $\varphi$.

Substituting approximate trajectory (159) and (160) into (95), it is easy to see that the pure source of twisted photons can be obtained when

$$
x_{+}(0) \approx 0, \quad v_{\perp}(0) \approx 0,
$$

i.e., in the strongly degenerate case. In this paper, we will investigate only this case. The plots of typical trajectories in this case are presented in Fig. 4. The calculations are performed along the same lines as those made above, except that the shift $\xi \rightarrow \xi+T N / 2$ is unnecessary. In particular, formulas (125) and (126) hold, and the radiation amplitude is proportional to

$$
\begin{aligned}
I_{3}+\frac{1}{2}\left(I_{+}+I_{-}\right)= & \frac{1}{2} \sum_{n=-\infty}^{\infty} j_{m-n}\left(k_{\perp} x_{+}(0), k_{\perp} x_{-}(0)\right) e^{i n \varphi_{0}} \\
& \times \int_{0}^{T N} d \xi g_{n}(\xi) e^{-i \xi x_{n}(\xi)}
\end{aligned}
$$

On stretching the variable $\xi \rightarrow T N \xi$, it is clear that the integral over $\xi$ can be approximately evaluated by using the saddle point approximation. For the envelope with one maximum as, for example,

$$
f(\xi)=f_{0} \sin ^{2}(\Omega \xi /(2 N)),
$$

the function $\xi x_{n}(\xi)$ has two extrema, $\xi_{ \pm}\left(k_{0}\right)$, on the interval $(0, T N)$, in a general position. At these extrema,

$k_{0}^{n}=\frac{2 \Omega n v_{-}^{2}}{\left(1-\zeta n_{3}\right)\left(1+K^{2}+2 \xi K K^{\prime}\right)+\left(1+\zeta n_{3}\right) v_{-}^{2}}>0$.

Therefore, we have

$$
\begin{aligned}
I_{3}+\frac{1}{2}\left(I_{+}+I_{-}\right) \approx & \sqrt{\frac{\pi}{2}} \sum_{n=-\infty}^{\infty} j_{m-n}\left(k_{\perp} x_{+}(0), k_{\perp} x_{-}(0)\right) e^{i n \varphi_{0}} \\
& \times\left[\left.\frac{g_{n}(\xi) e^{-i \xi x_{n}(\xi)}}{\sqrt{i\left(\xi x_{n}(\xi)\right)^{\prime \prime}}}\right|_{\xi=\xi_{+}}+\left.\frac{g_{n}(\xi) e^{-i \xi x_{n}(\xi)}}{\sqrt{i\left(\xi x_{n}(\xi)\right)^{\prime \prime}}}\right|_{\xi=\xi_{-}}\right],
\end{aligned}
$$

where the principal branch of the square root is taken. The contribution of the boundaries is suppressed since, in the leading order in $1 / N$, the same contribution but with opposite sign comes from the edge radiation (see, for details, e.g., Refs. $[98,100])$. As a result, the contributions of the internal stationary points are only relevant. One of the extremum points, $\xi_{+}$, is close to the point where $f^{\prime}(\xi)=0$; i.e., $K(\xi)$ is close to its maximum value at this point. Taking into account the form of $g_{n}(\xi)$, we see that this stationary point gives the leading contribution to (165). The main maximum is located approximately at

$k_{0}^{n} \approx \frac{2 \Omega n v_{-}^{2}}{\left(1-\zeta n_{3}\right)\left(1+K_{\max }^{2}\right)+\left(1+\zeta n_{3}\right) v_{-}^{2}}, \quad \Omega n>0$.

In fact, the maximum is slightly shifted to the right since the stationary point $\xi_{+}$is displaced a little from the extremum of $K(\xi)$. If $k_{\perp}\left|x_{+}(0)\right| \ll 1$, then $j_{m-n}\left(k_{\perp} x_{+}(0), k_{\perp} x_{-}(0)\right)=\delta_{m n}$, and 

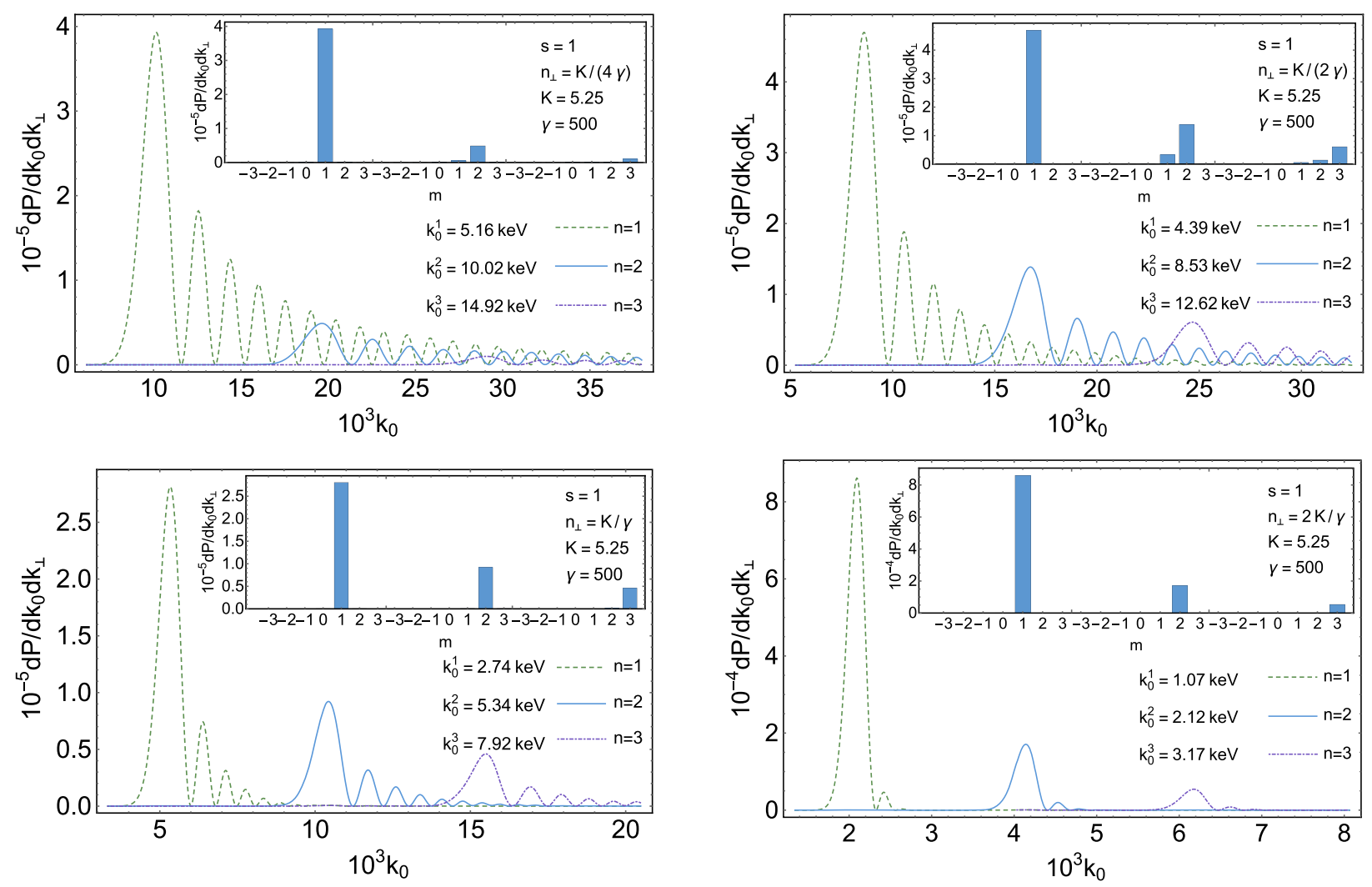

FIG. 5. The radiation of twisted photons in head-on collision of $256 \mathrm{MeV}$ electrons with the circularly polarized electromagnetic wave produced by the $\mathrm{CO}_{2}$ laser with the wavelength $10 \mu \mathrm{m}$, intensity $10^{18} \mathrm{~W} / \mathrm{cm}^{2}$, and amplitude envelope (163) with $N=20$. These data correspond to $\Omega \approx 2.8 \times 10^{-7}$ and $f_{0} \approx 1.47 \times 10^{-6}$. The first three harmonics (166) are depicted. The applicability conditions (84) are satisfied for $\sigma_{\perp} \lesssim 10^{3} m_{e}^{-1}$. The photon energy is measured in the electron rest energies. Insets: The distribution over $m$ at the main maximum of harmonics.

$$
\begin{aligned}
d P\left(s, m, k_{\perp}, k_{3}\right)= & e^{2}\left|\frac{g_{m}(\xi) e^{-i \xi x_{m}(\xi)}}{\sqrt{i\left(\xi x_{m}(\xi)\right)^{\prime \prime}}}\right|_{\xi=\xi_{+}} \\
& +\left.\left.\frac{g_{m}(\xi) e^{-i \xi x_{m}(\xi)}}{\sqrt{i\left(\xi x_{m}(\xi)\right)^{\prime \prime}}}\right|_{\xi=\xi_{-}}\right|^{2} n_{\perp}^{3} \frac{d k_{3} d k_{\perp}}{32 \pi} .
\end{aligned}
$$

At the extremum points, $\xi \sim N, x_{n}^{\prime}(\xi) \sim 1 / N$, and $x_{n}^{\prime \prime}(\xi) \sim$ $1 / N^{2}$. Therefore, the radiation probability is proportional to $N$.

The dependence of the probability density on $k_{0}$ differs from the profile $\delta_{N}^{2}\left(x_{m}\right)$ and is depicted in Figs. 5, 6, and 7. As is seen, the radiation probability $d P(m)$ is nearly zero for $k_{0}<k_{0}^{m}$; then, it rapidly grows in the vicinity of $k_{0}=k_{0}^{m}$, and for $k_{0}>k_{0}^{m}$, it declines to zero and oscillates. When $n_{\perp} \gamma \lesssim K_{\max } / 2$, this decrease is quite slow. So, for these values of $n_{\perp}$, the radiation probability $d P\left(m, k_{0}\right)$ taken in the neighborhood of the point $k_{0}=k_{0}^{m}$ can contain a considerable contribution of photons with the projection of the total angular momentum $m-1$. For very small $n_{\perp}$, the contribution of photons with all the lower projections of the angular momentum are relevant (see Figs. 5 and 6). When $n_{\perp} \gamma \approx K_{\max }$, the peaks of $d P\left(m, k_{0}\right)$ with different $m$ are well separated. In that case, the radiation at $k_{0} \approx k_{0}^{m}$ consists of twisted photons with the projection of the angular momentum $m$; i.e., the selection rule $m=\chi n$ is fulfilled, where $n$ is the number of harmonic (166) and $\chi= \pm 1$ is the handedness of the helix along which the electron is moving.

As follows from (73), most of twisted photons are radiated at lower harmonics (166). In the wiggler case, $K \gg 1$, these harmonics are fairly well described as the Lorentz-boosted lower harmonics of synchrotron radiation [see the discussion after (66)]. They would perfectly coincide if the charged particle moved along an ideal helix. Those lower harmonics were studied in Ref. [9], in which the effect of blossoming out rose was established; in the ultrarelativistic limit, these harmonics do not drive to the orbit plane, and the maximum intensity of radiation of every harmonic is achieved at some finite angle to the orbit plane. These angles for $\beta=1$ are given in Sec. 1.3.4 of Ref. [9]. The maxima of the first harmonic are located at the angles $\theta^{\prime}=\{0, \pi\}$.

In the laboratory frame, the imprint of this effect on the properties of radiation is as follows. One observes the 

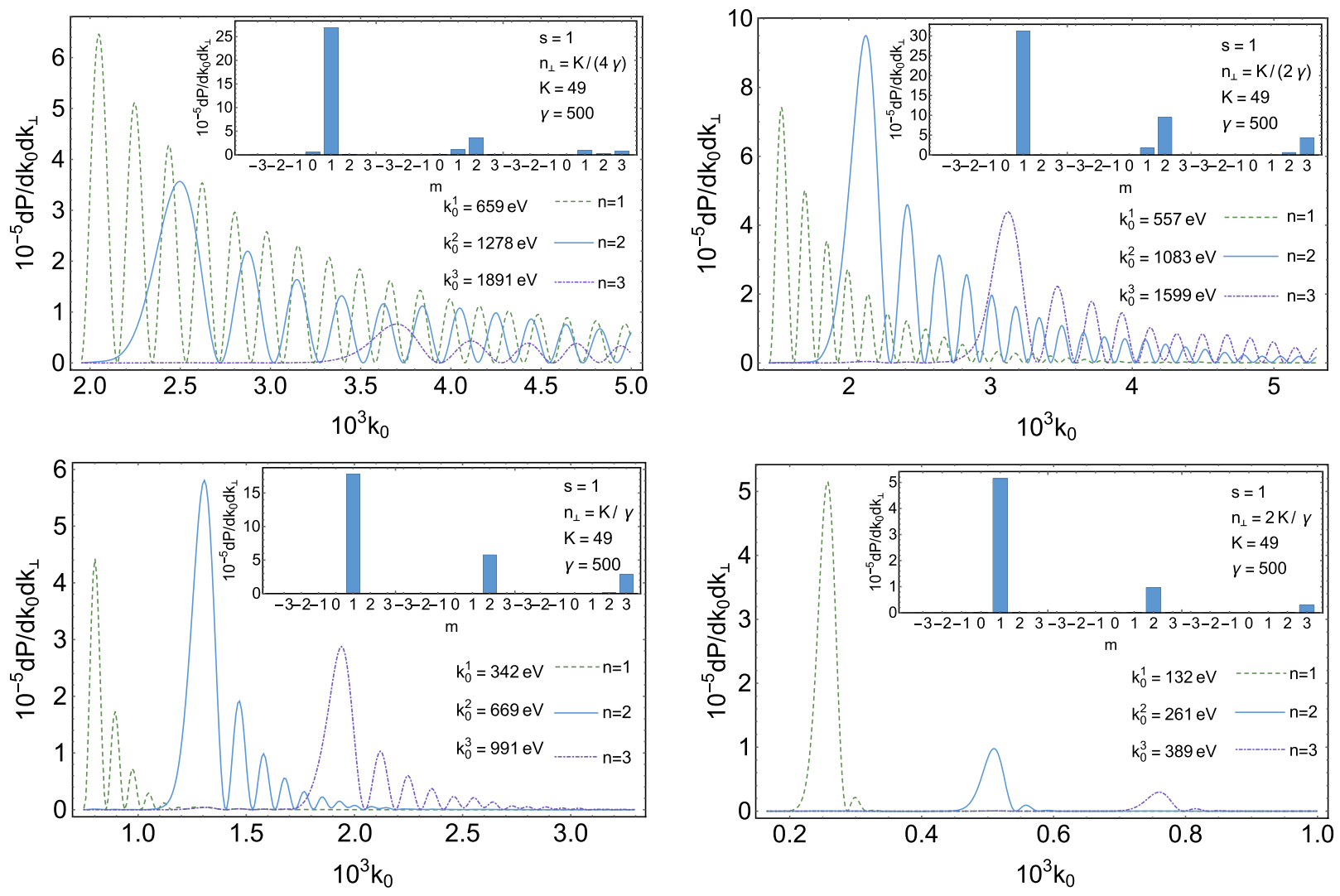

FIG. 6. The radiation of twisted photons in head-on collision of $256 \mathrm{MeV}$ electrons with the circularly polarized electromagnetic wave produced by the Ti:Sa laser with parameters (86) and amplitude envelope (163) with $N=20$. The first three harmonics (166) are depicted. The applicability conditions (84) are satisfied for $\sigma_{\perp} \lesssim 10^{3} m_{e}^{-1}$. The photon energy is measured in the electron rest energies. Insets: The distribution over $m$ at the main maximum of harmonics.

maxima of radiation of twisted photons at these harmonics at the angles taken from Ref. [9] and substituted into (67). The orbit plane is seen in the laboratory frame as a cone with opening $2 K / \gamma$. Since, in the synchrotron frame, the front lobes are mostly right-handed polarized (if, in the laboratory frame, the particle moves along a right-handed helix) and the back lobes are mostly left-handed polarized, and this property is Lorentz invariant, in the laboratory frame, the
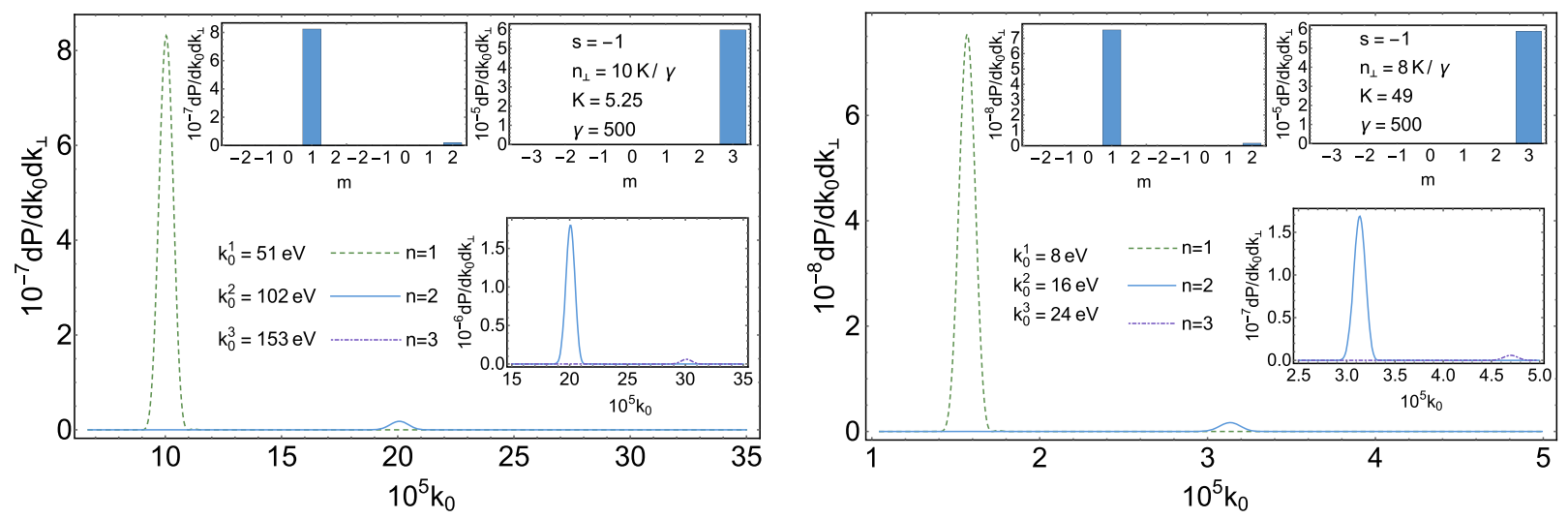

FIG. 7. The imprint of the blossoming out rose effect [9] on the radiation of twisted photons in head-on collision of electrons with circularly polarized electromagnetic wave produced by the $\mathrm{CO}_{2}$ (left panel) and Ti:Sa (right panel) lasers. The parameters are the same as in Figs. 5 and 6. The first harmonic (166) with $s=-1$ dominates. This is just a Lorentz-boosted back lobe of the first harmonic of synchrotron radiation (see Sec. 1.3.4 of Ref. [9]). It is mostly left-handed polarized, and this property is preserved by the Lorentz transformations. This harmonic does not die out even for $n_{\perp} \approx 1$. Upper left insets: The distributions over $m$ at the main maxima of the first two harmonics. Upper right insets: The distribution over $m$ at the main maximum of the third harmonic. Lower insets: The second and third harmonics are separately depicted. 
twisted photons with $s=1$ dominate for $n_{\perp}<K / \gamma$, while for $n_{\perp}>K / \gamma$, the twisted photons with $s=-1$ prevail. The first harmonic with $s=-1$ does not die out for large $n_{\perp}$ and even for $n_{\perp} \approx 1$. The plots of lower harmonics are presented in Fig. 7. Strictly speaking, formula (38) is not applicable for so large $n_{\perp}$. However, in the case of small quantum recoil, we can use exact formula (36) in Ref. [33]. Numerical calculation shows that Eq. (38) of the present paper and Eq. (36) in Ref. [33] give the same results in this case.

\section{CONCLUSION}

Let us summarize the results. Using the BK semiclassical approach [1-4], we derived the general formula for the one-photon radiation probability of a twisted photon by scalar (32) and Dirac (38) ultrarelativistic particles moving in the electromagnetic field of a general configuration. This formula takes into account the quantum recoil undergone by a charged particle in radiating the twisted photon and, in the case of negligible recoil, turns into the formula given in Ref. [33]. It also gives the average number of photons in the out state and the probability of inclusive process (1) in the first Born approximation. Then, we applied this formula to radiation of charged particles in helical undulators and in circularly polarized laser waves with a plane wave front. The explicit formulas for the probability to record the twisted photon by a detector were obtained in these cases. The inclusion of quantum recoil forbids radiation of twisted photons with energies larger than the initial particle energy. We established that, as a rule, the quantum recoil increases the total yield of radiation in comparison with classical formulas (see Fig. 2) and, at the same time, diminishes the energy of radiated photons. The spin degrees of freedom of a radiating particle increase the probability of radiation of twisted photons.

The conditions when the developed semiclassical approach is justified were found and analyzed. The most stringent among these conditions is (17). It guarantees that, in describing the radiation of twisted photons, it is sufficient to characterize the particle wave packet by its average coordinate and momentum. In particular, it turns out that the radiation of twisted photons with large projection $m$ of the total angular momentum produced by electrons in helical wigglers and strong laser waves can be described semiclassically only in the case of a small quantum recoil. In the dipole regime, the quantum recoil can be substantial and still be described semiclassically (see Fig. 2). We found estimate (73) for the number of twisted photons with large projections of the total angular momentum produced in the forward radiation. We also described the effect of the blossoming out rose [9] in the radiation of twisted photons by electrons evolving in strong laser waves with circular polarization and wigglers (see Fig. 7).

For an example, we considered the radiation of twisted photons with large angular momentum in the helical wiggler (see Fig. 1) and in the circularly polarized strong laser waves produced by the $\mathrm{CO}_{2}$ and Ti:Sa lasers (Figs. 3, 5,6 , and 7). The parameters are given in these figures. In particular, we showed that mega-electron-volt twisted photons with $m \sim 5$ can be generated in helical wigglers. As for lasers, we found that the design of a sufficiently pure source of twisted photons based on the nonlinear Compton process is only possible for long laser pulses, $N \gtrsim 10$, with a smooth amplitude envelope. For short pulses, the escape direction of a twisted photon depends severely on the initial phase of a laser wave that is virtually uncontrollable. Therefore, the detector (the atom, for example) will feel the radiation consisting of twisted photons with a wide spread of the total angular momentum projections $m$ (see Fig. 3). For the systems concerned, we also described the effect of a finite width of a particle bunch on the incoherent radiation of twisted photons [77].

\section{ACKNOWLEDGMENTS}

We are thankful to Yu. L. Pivovarov and D. V. Karlovets for fruitful conversations. This work is supported by the Russian Science Foundation (Project No. 17-72-20013).

\section{APPENDIX A: TWISTED PHOTONS IN TERMS OF PLANE WAVES}

For the convenience of the reader, we shall provide the representation of the states of twisted photons in terms of the plane wave ones (see the detailed exposition, e.g., in Ref. [25]). The states describing the photons with plane wave front,

$$
\left|s, k_{1}, k_{2}, k_{3}\right\rangle
$$

and the states of twisted photons,

$$
\left|s, m, k_{\perp}, k_{3}\right\rangle,
$$

constitute the complete sets in the Hilbert space of oneparticle states:

$$
\sum_{s= \pm 1} \int \frac{V d \mathbf{k}}{(2 \pi)^{3}} 2 k_{0}\left|s, k_{1}, k_{2}, k_{3}\right\rangle\left\langle s, k_{1}, k_{2}, k_{3}\left|=\sum_{s= \pm 1} \sum_{m=-\infty}^{\infty} \int_{-\infty}^{\infty} \frac{L_{3} d k_{3}}{2 \pi} \int_{0}^{\infty} \frac{R d k_{\perp}}{\pi} 2 k_{0}\right| s, m, k_{\perp}, k_{3}\right\rangle\left\langle s, m, k_{\perp}, k_{3}\right|=1 .
$$

The states are normalized as 


$$
\begin{aligned}
\left\langle s, k_{1}, k_{2}, k_{3} \mid s, k_{1}, k_{2}, k_{3}\right\rangle & =\left\langle s, m, k_{\perp}, k_{3} \mid s, m, k_{\perp}, k_{3}\right\rangle \\
& =\left(2 k_{0}\right)^{-1},
\end{aligned}
$$

where $k_{0}=|\mathbf{k}|=\sqrt{k_{\perp}^{2}+k_{3}^{3}}$. One can decompose the state (A2) in terms of the states (A1). Carrying out rather simple calculations, we come to

$$
\begin{aligned}
\left|s, m, k_{\perp}, k_{3}\right\rangle= & \frac{\sqrt{2 k_{0} V}}{2 \sqrt{R L_{3}}}\left(\frac{k_{\perp}}{k_{0}}\right)^{1 / 2} \\
& \times \int_{-\pi}^{\pi} \frac{d \varphi}{2 \pi} i^{-m} e^{i m \varphi}\left|s, k_{\perp} \cos \varphi, k_{\perp} \sin \varphi, k_{3}\right\rangle .
\end{aligned}
$$

Obviously, the states describing twisted photons depend on the choice of the detector axis (the axis 3) and transform nontrivially under translations perpendicular to this axis. Such a dependence is well known and was measured experimentally (see, e.g., Refs. [39,43,102-106]).

\section{APPENDIX B: ESTIMATES OF THE WAVE PACKET WAIST}

The estimate (17) is the most stringent condition on the range of applicability of formula (32). It is absent in the semiclassical description of radiation of plane wave photons [4]. The narrower the transverse size of a particle wave packet is, the better this condition is satisfied. Currently, there are techniques allowing one to produce the electron wave packets with the transverse size of order $1 \AA$ [107]. For a particle, which is at rest on average, the width of the wave packet cannot be smaller than the Compton wavelength. Otherwise, the momentum uncertainty is larger than $2 m$, and the height of a potential barrier confining such a particle must be larger than $2 \mathrm{~m}$. Such fields create electron-positron pairs, and the problem becomes essentially multiparticle. Nevertheless, if the electron is moving on average, then its wave packet can be squeezed more strongly in the transverse directions with the aid of the fields not exceeding the critical (Schwinger) field in the laboratory frame. In the comoving reference frame, the potential well depth can become larger than the critical one, but this does not result in pair creation. In passing to the comoving frame, the magnetic field arises, and the respective invariants of the electromagnetic field remain the same as in the laboratory frame.

This situation is naturally realized for axial channeling of particles in crystals (see for details, e.g., Refs. $[4,8,9,89,108]$ ). Suppose that the particle momentum component $p_{3} \gg m_{e}$ and $\left|p_{1,2}\right| \ll p_{3}$. Then, the solution of the stationary Dirac or Klein-Gordon equations is reduced approximately to the solution of the Schrödinger equation with a certain effective potential $U\left(\mathbf{x}_{\perp}\right)$,

$$
\left(\frac{\mathbf{p}_{\perp}^{2}}{2 m_{\mathrm{eff}}}+U\right) \psi=\delta E \psi
$$

where $\delta E=E-m_{\mathrm{eff}}, m_{\mathrm{eff}}^{2}:=m_{e}^{2}+p_{3}^{2}$, and it is assumed that $|U| \ll m_{\text {eff }}$ and $|\delta E| \ll m_{\text {eff }}$. The effective mass is well approximated by

$$
m_{\mathrm{eff}} \approx \gamma m_{e} .
$$

The potential barrier of the height $U_{0}$ can hold a particle with typical momentum uncertainty

$$
\left|\Delta p_{\perp}\right| \lesssim \sqrt{2 m_{\text {eff }} U_{0}}
$$

Then, from the uncertainty relation, we obtain the minimum transverse size

$$
\sigma_{\perp}^{m} \sim\left(2 m_{\mathrm{eff}} U_{0}\right)^{-1 / 2} \approx 1 /\left(\theta_{c} \varepsilon\right)
$$

where the critical Lindhard angle has been introduced:

$$
\theta_{c}:=\sqrt{\frac{2 U_{0}}{\varepsilon}}, \quad U_{0} \sim Z \alpha^{2} m_{e} .
$$

Since $\beta_{\perp} \approx \theta_{c}$, then

$$
\sigma_{\perp}^{m} \sim 1 /\left(m_{e} K\right) .
$$

As we see, the transverse size of the particle wave packet can be much smaller than the Compton wavelength for axal channeling in the wiggler regime $[109,110]$. The maximum transverse squeezing of a particle wave packet is achieved at

$$
\sigma_{\perp}^{m} \sim 1 /\left(m_{e} \gamma\right)
$$

However, this is possible only in the external fields of the same order as the critical one in the laboratory frame. Such maximally localized wave packets were investigated in Refs. [111,112].

Consider the fulfillment of condition (17) for axial channeling with the wave packet transverse size (B6). The respective undulator frequency is estimated as

$$
\omega \sim \frac{\pi \theta_{c}}{d},
$$

where $d$ is the channel width,

$$
d \sim 1 \AA \sim 10^{3} m_{e}^{-1} .
$$

Then, in the dipole regime, at the $n$th harmonic

$$
k_{0} \sim 2 \omega \gamma^{2} n .
$$

Hence, 


$$
k_{\perp} \sigma_{\perp}^{m} \sim 2 \pi \frac{n_{\perp} \gamma}{m_{e} d} n \ll 1
$$

Bearing in mind that $n_{\perp} \gamma \lesssim 1$, we see that (17) is satisfied. This condition is fulfilled even for $\sigma_{\perp}=d$ in the dipole regime for not-too-large harmonic numbers. In the wiggler case for $n_{\perp} \gamma \approx K$, we have

$$
k_{0} \sim \omega \gamma^{2} n / K^{2} .
$$

Then,

$$
k_{\perp} \sigma_{\perp}^{m} \sim \frac{\pi n}{m_{e} d K} \ll 1 .
$$

In fact, condition (17) is already satisfied for $\sigma_{\perp} n \lesssim d / 10$. The energy shift due to quantum recoil was neglected in these formulas. The inclusion of quantum recoil only improves the estimate.

The dynamical picture looks as follows. The wide particle wave packet falls onto the crystal surface and, in the channeling regime, is split into wave packets with transverse sizes from (B6) up to $d$. Because of interaction with the crystalline potential and other electrons, the different parts of the wave packet moving in different channels do not almost interfere. This picture, of course, agrees with the quantum channeling theory where the states of electrons are usually described by Bloch waves $[4,8]$. In our case, if the particle is in channeling regime, the overlap integrals are small, and so the parts of the electron wave packet do not virtually interfere. To describe the radiation of twisted photons produced by each part of such dispersed wave packet, Eq. (38) can be used. As the particle escaped the crystal, the parts of the wave packet spread in the transverse directions with the characteristic velocity $\beta_{\perp} \approx \theta_{c}$.

\section{APPENDIX C: EVALUATION OF INTEGRALS OVER THE AZIMUTH ANGLE}

It is convenient to evaluate the integrals over the azimuth angles of the vectors $\mathbf{k}_{1,2}$ in expression (37) as follows. Up to a common factor, which can be restored easily from (A5), we have the correspondence

$$
\begin{aligned}
& \mathbf{f}_{1}^{*} \rightarrow\left(\frac{1}{2}\left[a_{+}^{*} \mathbf{e}_{+}+a_{-}^{*} \mathbf{e}_{-}\right]+a_{3}^{*} \mathbf{e}_{3}\right) e^{-i k_{3} q_{1} x_{13}}=: \mathbf{a}_{1}^{*}, \\
& \mathbf{f}_{2} \rightarrow\left(\frac{1}{2}\left[a_{+} \mathbf{e}_{-}+a_{-} \mathbf{e}_{+}\right]+a_{3} \mathbf{e}_{3}\right) e^{i k_{3} q_{2} x_{23}}=: \mathbf{a}_{2} .
\end{aligned}
$$

Hereinafter, for brevity, we write only those arguments of the mode functions $a_{ \pm, 3}, a_{ \pm, 3}^{*}$ that differ from those written in Eq. (39). The basis vectors $\mathbf{e}_{i}$ are defined in Eq. (9) of Ref. [33]. Notice that $q_{1,2}=q_{i}$ in (37), but we keep $q_{1,2}$ different. Then, for example,

$$
\left(\mathbf{f}_{1}^{*} \dot{\mathbf{x}}_{1}\right)\left(\mathbf{f}_{2} \dot{\mathbf{x}}_{2}\right) \rightarrow e^{i k_{3}\left(q_{2} x_{23}-q_{1} x_{13}\right)}\left(\frac{1}{2}\left[\dot{x}_{1-} a_{-}^{*}+\dot{x}_{1+} a_{+}^{*}\right]+\dot{x}_{13} a_{3}^{*}\right)\left(\frac{1}{2}\left[\dot{x}_{2+} a_{-}+\dot{x}_{2-} a_{+}\right]+\dot{x}_{23} a_{3}\right)=\left(\mathbf{a}_{1}^{*} \dot{\mathbf{x}}_{1}\right)\left(\mathbf{a}_{2} \dot{\mathbf{x}}_{2}\right)
$$

The additional powers of $\mathbf{k}_{1,2}$ can be obtained by differentiation of the expression with respect to

$$
\mathbf{b}_{1,2}:=q_{1,2} x_{31,2} \mathbf{e}_{3}+\mathbf{x}_{\perp 1,2} \text {. }
$$

For example,

$$
k_{0}\left(\mathbf{f}_{2} \mathbf{f}_{1}^{*}\right)\left(\dot{\mathbf{x}}_{2} \mathbf{k}_{1}\right) \rightarrow i k_{0}\left(\dot{x}_{23} \frac{\partial}{\partial b_{13}}+\dot{x}_{2+} \frac{\partial}{\partial b_{1+}}+\dot{x}_{2-} \frac{\partial}{\partial b_{1-}}\right)\left(\mathbf{a}_{1}^{*} \mathbf{a}_{2}\right) .
$$

The derivatives of the mode functions are calculated with the aid of relations (A3) in Ref. [33]. They read as

$$
\begin{aligned}
& \frac{\partial \mathbf{a}_{2}}{\partial b_{2+}}=\frac{k_{\perp}}{2} \mathbf{a}_{2}(m-1), \quad \frac{\partial \mathbf{a}_{2}}{\partial b_{2-}}=-\frac{k_{\perp}}{2} \mathbf{a}_{2}(m+1), \quad \frac{\partial \mathbf{a}_{2}}{\partial b_{23}}=i k_{3} \mathbf{a}_{2}, \\
& \frac{\partial \mathbf{a}_{1}^{*}}{\partial b_{1+}}=-\frac{k_{\perp}}{2} \mathbf{a}_{1}^{*}(m+1), \quad \frac{\partial \mathbf{a}_{1}^{*}}{\partial b_{1-}}=\frac{k_{\perp}}{2} \mathbf{a}_{1}^{*}(m-1), \quad \frac{\partial \mathbf{a}_{1}^{*}}{\partial b_{13}}=-i k_{3} \mathbf{a}_{1}^{*} .
\end{aligned}
$$

Applying these relations to the expression in the square brackets in (37), we arrive at the rather bulky formula 


$$
\begin{aligned}
( & \left.P_{01}+P_{01}^{\prime}\right)\left(P_{02}+P_{02}^{\prime}\right)\left(\mathbf{a}_{1}^{*} \dot{\mathbf{x}}_{1}\right)\left(\mathbf{a}_{2} \dot{\mathbf{x}}_{2}\right)+k_{0}^{2}\left[\left(\mathbf{a}_{1}^{*} \mathbf{a}_{2}\right)\left(\dot{\mathbf{x}}_{1}-n_{3} \mathbf{e}_{3}, \dot{\mathbf{x}}_{2}-n_{3} \mathbf{e}_{3}\right)-\left(\mathbf{a}_{1}^{*}, \dot{\mathbf{x}}_{2}-n_{3} \mathbf{e}_{3}\right)\left(\mathbf{a}_{2}, \dot{\mathbf{x}}_{1}-n_{3} \mathbf{e}_{3}\right)\right] \\
& +\frac{i k_{0} k_{\perp}}{2}\left[\left(\mathbf{a}_{1}^{*}(m-1),\left(\dot{\mathbf{x}}_{2}-n_{3} \mathbf{e}_{3}\right) a_{2-}-\dot{x}_{2-} \mathbf{a}_{2}\right)-\left(\mathbf{a}_{1}^{*}(m+1),\left(\dot{\mathbf{x}}_{2}-n_{3} \mathbf{e}_{3}\right) a_{2+}-\dot{x}_{2+} \mathbf{a}_{2}\right)\right. \\
& \left.-\left(\left(\dot{\mathbf{x}}_{1}-n_{3} \mathbf{e}_{3}\right) a_{1-}^{*}-\dot{x}_{1+} \mathbf{a}_{1}^{*}, \mathbf{a}_{2}(m-1)\right)+\left(\left(\dot{\mathbf{x}}_{1}-n_{3} \mathbf{e}_{3}\right) a_{1+}^{*}-\dot{x}_{1-} \mathbf{a}_{1}^{*}, \mathbf{a}_{2}(m+1)\right)\right] \\
& +\frac{k_{\perp}^{2}}{4}\left[a_{1-}^{*}(m+1) a_{2+}(m-1)+a_{1+}^{*}(m-1) a_{2-}(m+1)-a_{1+}^{*}(m+1) a_{2+}(m+1)\right. \\
& \left.-a_{1-}^{*}(m-1) a_{2-}(m-1)+2\left(\mathbf{a}_{1}^{*}(m+1), \mathbf{a}_{2}(m+1)\right)+2\left(\mathbf{a}_{1}^{*}(m-1), \mathbf{a}_{2}(m-1)\right)\right],
\end{aligned}
$$

where $n_{3}=k_{3} / k_{0}$. Now, take into account that, in the region where the radiation of an ultrarelativistic particle is concentrated,

$$
k_{0} / \varepsilon \lesssim 1, \quad\left|\dot{x}_{ \pm}\right| \sim \varkappa / \gamma, \quad\left|\dot{x}_{3}\right| \approx 1, \quad\left|\dot{x}_{3}-n_{3}\right| \lesssim \varkappa^{2} / \gamma^{2}, \quad\left|n_{\perp}\right| \lesssim \varkappa / \gamma, \quad n_{3} \approx 1,
$$

where $n_{\perp}=k_{\perp} / k_{0}$. It follows from the explicit expressions for the mode functions that

$$
\left|a_{ \pm}^{*}\right| \sim\left|a_{ \pm}\right| \lesssim \gamma / \varkappa, \quad\left|a_{3}\right| \sim\left|a_{ \pm}^{*} a_{\mp}\right| \sim 1 .
$$

Expanding the scalar products in (C6) and neglecting the terms of order $\varkappa / \gamma \ll 1$ in comparison with the main contribution, we obtain

$$
\begin{aligned}
& e^{i k_{3}\left(b_{23}-b_{13}\right)}\left\{\left(P_{01}+P_{01}^{\prime}\right)\left(P_{02}+P_{02}^{\prime}\right)\left(\frac{1}{2}\left[\dot{x}_{1-} a_{-}^{*}+\dot{x}_{1+} a_{+}^{*}\right]+\dot{x}_{13} a_{3}^{*}\right)\left(\frac{1}{2}\left[\dot{x}_{2+} a_{-}+\dot{x}_{2-} a_{+}\right]+\dot{x}_{23} a_{3}\right)\right. \\
& \left.\quad+\frac{k_{0}^{2}}{4}\left[\left(\dot{x}_{1+} a_{+}^{*}-i n_{\perp} a_{+}^{*}(m-1)\right)\left(\dot{x}_{2-} a_{+}+i n_{\perp} a_{+}(m-1)\right)+\left(\dot{x}_{1-} a_{-}^{*}+i n_{\perp} a_{-}^{*}(m+1)\right)\left(\dot{x}_{2+} a_{-}-i n_{\perp} a_{-}(m+1)\right)\right]\right\} .
\end{aligned}
$$

Setting $q_{1,2}=q_{i}$ and taking into account the common factor in (37), we deduce (38).

[1] V. N. Baier and V.M. Katkov, Quasiclassical theory of bremsstrahlung by relativistic particles, Zh. Eksp. Teor. Fiz. 55, 1542 (1968) [Sov. Phys. JETP 28, 807 (1969)].

[2] V. N. Baier, V. M. Katkov, A. I. Mil'shtein, and V. M. Strakhovenko, The theory of quantum processes in the field of a strong electromagnetic wave, Zh. Eksp. Teor. Fiz. 69, 783 (1975) [Sov. Phys. JETP 42, 400 (1975)].

[3] V. N. Baier, V. M. Katkov, and V. M. Strakhovenko, Semiclassical theory of electromagnetic processes in a plane wave and a constant field, Zh. Eksp. Teor. Fiz. 100, 1713 (1991) [Sov. Phys. JETP 73, 945 (1991)].

[4] V. N. Baier, V.M. Katkov, and V.M. Strakhovenko, Electromagnetic Processes at High Energies in Oriented Single Crystals (World Scientific, Singapore, 1998).

[5] A. I. Akhiezer and N. F. Shul'ga, Quasiclassical theory of radiation emission from high-energy particles in an external field and the problem of boundary conditions, $\mathrm{Zh}$. Eksp. Teor. Fiz. 100, 791 (1991) [Sov. Phys. JETP 73, 437 (1991)].

[6] V. G. Bagrov, V. V. Belov, and A. Yu. Trifonov, Theory of spontaneous radiation by electrons in a trajectory-coherent approximation, J. Phys. A 26, 6431 (1993).
[7] V. V. Belov, D. V. Boltovskiy, and A. Yu. Trifonov, Theory of spontaneous radiation by bosons in quasi-classical trajectory-coherent approximation, Int. J. Mod. Phys. B 08, 2503 (1994).

[8] A. I. Akhiezer and N. F. Shulga, High-Energy Electrodynamics in Matter (Gordon and Breach, New York, 1996).

[9] V. G. Bagrov, G. S. Bisnovatyi-Kogan, V. A. Bordovitsyn, A. V. Borisov, O. F. Dorofeev, V. Y. Epp, V. S. Gushchina, and V. C. Zhukovskii, Synchrotron Radiation Theory and its Development (World Scientific, Singapore, 1999).

[10] V. G. Bagrov, V. V. Belov, and A. Yu. Trifonov, Methods of Mathematical Physics: Asymptotic Methods in Relativistic Quantum Mechanics (Tomsk Polytechnic University Press, Tomsk, 2006) [in Russian].

[11] V. Guidi, L. Bandiera, and V. Tikhomirov, Radiation generated by single and multiple volume reflection of ultrarelativistic electrons and positrons in bent crystals, Phys. Rev. A 86, 042903 (2012).

[12] V. G. Bezchastnov, A. V. Korol, and A. V. Solov'yov, Radiation from multi-GeV electrons and positrons in periodically bent silicon crystal, J. Phys. B 47, 195401 (2014). 
[13] L. Bandiera, E. Bagli, V. Guidi, and V. V. Tikhomirov, RADCHARM++: A C++ routine to compute the electromagnetic radiation generated by relativistic charged particles in crystals and complex structures, Nucl. Instrum. Methods Phys. Res., Sect. B 355, 44 (2015).

[14] T. N. Wistisen, A. Di Piazza, H. V. Knudsen, and U. I. Uggerhøj, Experimental evidence of quantum radiation reaction in aligned crystals, Nat. Commun. 9, 795 (2018).

[15] A. Di Piazza, C. Müller, K. Z. Hatsagortsyan, and C. H. Keitel, Extremely high-intensity laser interactions with fundamental quantum systems, Rev. Mod. Phys. 84, 1177 (2012).

[16] T. N. Wistisen, Interference effect in nonlinear Compton scattering, Phys. Rev. D 90, 125008 (2014).

[17] V. Dinu, C. Harvey, A. Ilderton, M. Marklund, and G. Torgrimsson, Quantum Radiation Reaction: From Interference to Incoherence, Phys. Rev. Lett. 116, 044801 (2016).

[18] J. L. Martins, M. Vranic, T. Grismayer, J. Vieira, R. A. Fonseca, and L. O. Silva, Modelling radiation emission in the transition from the classical to the quantum regime, Plasma Phys. Controlled Fusion 58, 014035 (2016).

[19] T. G. Blackburn, D. Seipt, S. S. Bulanov, and M. Marklund, Benchmarking semiclassical approaches to strong-field QED: Nonlinear Compton scattering in intense laser pulses, Phys. Plasmas 25, 083108 (2018).

[20] J. M. Cole et al., Experimental Evidence of Radiation Reaction in the Collision of a High-Intensity Laser Pulse with a Laser-Wakefield Accelerated Electron Beam, Phys. Rev. X 8, 011020 (2018).

[21] K. Poder et al., Experimental Signatures of the Quantum Nature of Radiation Reaction in the Field of an Ultraintense Laser, Phys. Rev. X 8, 031004 (2018).

[22] Y.-F. Li, R. Shaisultanov, K. Z. Hatsagortsyan, F. Wan, C. H. Keitel, and J.-X. Li, Ultrarelativistic Electron Beam Polarization in Single-Shot Interaction with an Ultraintense Laser Pulse, Phys. Rev. Lett. 122, 154801 (2019).

[23] G. Molina-Terriza, J. P. Torres, and L. Torner, Twisted photons, Nat. Phys. 3, 305 (2007).

[24] U. D. Jentschura and V. G. Serbo, Generation of HighEnergy Photons with Large Orbital Angular Momentum by Compton Backscattering, Phys. Rev. Lett. 106, 013001 (2011).

[25] U. D. Jentschura and V. G. Serbo, Compton upconversion of twisted photons: Backscattering of particles with nonplanar wave functions, Eur. Phys. J. C 71, 1571 (2011).

[26] I. P. Ivanov, Colliding particles carrying nonzero orbital angular momentum, Phys. Rev. D 83, 093001 (2011).

[27] B. A. Knyazev and V. G. Serbo, Beams of photons with nonzero projections of orbital angular momenta: New results, Phys. Usp. 61, 449 (2018).

[28] M. J. Padgett, Orbital angular momentum 25 years on, Opt. Express 25, 11265 (2017).

[29] H. Rubinsztein-Dunlop et al., Roadmap on structured light, J. Opt. 19, 013001 (2017).

[30] The Angular Momentum of Light, edited by D. L. Andrews and M. Babiker (Cambridge University Press, New York, 2013).

[31] Twisted Photons, edited by J.P. Torres and L. Torner (Wiley-VCH, Weinheim, 2011).
[32] Structured Light and Its Applications, edited by D. L. Andrews (Academic, Amsterdam, 2008).

[33] O. V. Bogdanov, P. O. Kazinski, and G. Yu. Lazarenko, Probability of radiation of twisted photons by classical currents, Phys. Rev. A 97, 033837 (2018).

[34] L. D. Landau and E. M. Lifshitz, The Classical Theory of Fields (Pergamon, Oxford, 1962).

[35] J. D. Jackson, Classical Electrodynamics (Wiley, New York, 1962).

[36] J. Leach, M. J. Padgett, S. M. Barnett, S. Franke-Arnold, and J. Courtial, Measuring the Orbital Angular Momentum of a Single Photon, Phys. Rev. Lett. 88, 257901 (2002).

[37] G. C. G. Berkhout, M. P. J. Lavery, Johannes Courtial, M. W. Beijersbergen, and M. J. Padgett, Efficient Sorting of Orbital Angular Momentum States of Light, Phys. Rev. Lett. 105, 153601 (2010).

[38] T. Su, R. P. Scott, S. S. Djordjevic, N. K. Fontaine, D. J. Geisler, X. Cai, and S. J. B. Yoo, Demonstration of free space coherent optical communication using integrated silicon photonic orbital angular momentum devices, Opt. Express 20, 9396 (2012).

[39] M. P. J. Lavery, J. Courtial, and M. J. Padgett, Measurement of light's orbital angular momentum, in The Angular Momentum of Light, edited by D. L. Andrews and M. Babiker (Cambridge University Press, New York, 2013).

[40] G. Ruffato, M. Girardi, M. Massari, E. Mafakheri, B. Sephton, P. Capaldo, A. Forbes, and F. Romanato, A compact difractive sorter for high-resolution demultiplexing of orbital angular momentum beams, Sci. Rep. 8, 10248 (2018).

[41] B. Paroli, M. Siano, L. Teruzzi, and M. A. C. Potenza, Single-shot measurement of phase and topological properties of orbital angular momentum radiation through asymmetric lateral coherence, Phys. Rev. Accel. Beams 22, 032901 (2019).

[42] Y. Taira, T. Hayakawa, and M. Katoh, Gamma-ray vortices from nonlinear inverse Thomson scattering of circularly polarized light, Sci. Rep. 7, 5018 (2017).

[43] A. Afanasev, V. G. Serbo, and M. Solyanik, Radiative capture of cold neutrons by protons and deuteron photodisintegration with twisted beams, J. Phys. G 45, 055102 (2018).

[44] S. V. Abdrashitov, O. V. Bogdanov, P. O. Kazinski, and T. A. Tukhfatullin, Orbital angular momentum of channeling radiation from relativistic electrons in thin Si crystal, Phys. Lett. A 382, 3141 (2018).

[45] V. Epp, J. Janz, and M. Zotova, Angular momentum of radiation at axial channeling, Nucl. Instrum. Methods Phys. Res., Sect. B 436, 78 (2018).

[46] M. Katoh, M. Fujimoto, H. Kawaguchi, K. Tsuchiya, K. Ohmi, T. Kaneyasu, Y. Taira, M. Hosaka, A. Mochihashi, and Y. Takashima, Angular Momentum of Twisted Radiation from an Electron in Spiral Motion, Phys. Rev. Lett. 118, 094801 (2017).

[47] Y. Taira and M. Katoh, Generation of optical vortices by nonlinear inverse Thomson scattering at arbitrary angle interactions, Astrophys. J. 860, 45 (2018).

[48] Y.-Y. Chen, J.-X. Li, K. Z. Hatsagortsyan, and C. H. Keitel, $\gamma$-Ray Beams with Large Orbital Angular Momentum via 
Nonlinear Compton Scattering with Radiation Reaction, Phys. Rev. Lett. 121, 074801 (2018).

[49] X.-L. Zhu, T.-P. Yu, M. Chen, S.-M. Weng, and Z.-M. Sheng, Generation of $\mathrm{GeV}$ positron and $\gamma$-photon beams with controllable angular momentum by intense lasers, New J. Phys. 20, 083013 (2018).

[50] S. Sasaki and I. McNulty, Proposal for Generating Brilliant X-Ray Beams Carrying Orbital Angular Momentum, Phys. Rev. Lett. 100, 124801 (2008).

[51] E. Hemsing, A. Marinelli, S. Reiche, and J. Rosenzweig, Longitudinal dispersion of orbital angular momentum modes in high-gain free-electron lasers, Phys. Rev. ST Accel. Beams 11, 070704 (2008).

[52] E. Hemsing, A. Marinelli, and J. B. Rosenzweig, Generating Optical Orbital Angular Momentum in a High-Gain Free-Electron Laser at the First Harmonic, Phys. Rev. Lett. 106, 164803 (2011).

[53] J. Bahrdt, K. Holldack, P. Kuske, R. Müller, M. Scheer, and P. Schmid, First Observation of Photons Carrying Orbital Angular Momentum in Undulator Radiation, Phys. Rev. Lett. 111, 034801 (2013).

[54] E. Hemsing, A. Knyazik, M. Dunning, D. Xiang, A. Marinelli, C. Hast, and J. B. Rosenzweig, Coherent optical vortices from relativistic electron beams, Nat. Phys. 9, 549 (2013).

[55] M. Katoh et al., Helical phase structure of radiation from an electron in circular motion, Sci. Rep. 7, 6130 (2017).

[56] P. R. Ribič et al., Extreme-Ultraviolet Vortices from a FreeElectron Laser, Phys. Rev. X 7, 031036 (2017).

[57] A. I. Nikishov and V. I. Ritus, Quantum processes in the field of a plane electromagnetic wave and in a constant field. I, Zh. Eksp. Teor. Fiz. 46, 776 (1964) [Sov. Phys. JETP 19, 529 (1964)].

[58] V. I. Ritus, Quantum effects of the interaction of elementary particles with an intense electromagnetic field, J. Sov. Laser Res. 6, 497 (1985).

[59] E.S. Fradkin, D. M. Gitman, and S. M. Shvartsman, Quantum Electrodynamics with Unstable Vacuum (Springer, Berlin, 1991).

[60] D. Yu. Ivanov, G. L. Kotkin, and V. G. Serbo, Complete description of polarization effects in emission of a photon by an electron in the field of a strong laser wave, Eur. Phys. J. C 36, 127 (2004).

[61] F. Mackenroth, A. Di Piazza, and C. H. Keitel, Determining the Carrier-Envelope Phase of Intense Few-Cycle Laser Pulses, Phys. Rev. Lett. 105, 063903 (2010).

[62] A. Di Piazza, K. Z. Hatsagortsyan, and C. H. Keitel, Quantum Radiation Reaction Effects in Multiphoton Compton Scattering, Phys. Rev. Lett. 105, 220403 (2010).

[63] A. Ilderton and G. Torgrimsson, Scattering in plane-wave backgrounds: Infrared effects and pole structure, Phys. Rev. D 87, 085040 (2013).

[64] L. Guo-Hua, L. Qing-Zheng, T. Ai-Ping, and L. Ying-Jun, The polarization effect of a laser in multiphoton Compton scattering, Chin. Phys. B 23, 054103 (2014).

[65] S. Stock, A. Surzhykov, S. Fritzsche, and D. Seipt, Compton scattering of twisted light: Angular distribution and polarization of scattered photons, Phys. Rev. A 92, 013401 (2015).
[66] J. A. Sherwin, Theoretical study of the double Compton effect with twisted photons, Phys. Rev. A 95, 052101 (2017).

[67] A. P. Potylitsyn, A. M. Kolchuzhkin, M. N. Strikhanov, and S. A. Strokov, Photon spectrum and polarization for high conversion coefficient in the Compton backscattering process, Nucl. Instrum. Methods Phys. Res., Sect. B 402, 216 (2017).

[68] A. Di Piazza, M. Tamburini, S. Meuren, and C. H. Keitel, Implementing nonlinear Compton scattering beyond the local-constant-field approximation, Phys. Rev. A 98, 012134 (2018).

[69] K. Gottfried and T.-M. Yan, Quantum Mechanics: Fundamentals (Springer, New York, 2003).

[70] R. Jáuregui and S. Hacyan, Quantum-mechanical properties of Bessel beams, Phys. Rev. A 71, 033411 (2005).

[71] I. Bialynicki-Birula and Z. Bialynicka-Birula, Beams of electromagnetic radiation carrying angular momentum: The Riemann-Silberstein vector and the classical-quantum correspondence, Opt. Commun. 264, 342 (2006).

[72] O. Matula, A. G. Hayrapetyan, V. G. Serbo, A. Surzhykov, and S. Fritzsche, Atomic ionization of hydrogen-like ions by twisted photons: Angular distribution of emitted electrons, J. Phys. B 46, 205002 (2013).

[73] H. M. Scholz-Marggraf, S. Fritzsche, V. G. Serbo, A. Afanasev, and A. Surzhykov, Absorption of twisted light by hydrogenlike atoms, Phys. Rev. A 90, 013425 (2014).

[74] W. Greiner, B. Müller, and J. Rafelski, Quantum Electrodynamics of Strong Fields (Springer, Heidelberg, 1985).

[75] A. B. Migdal, O. A. Markin, and I. I. Mishustin, The pion spectrum in nuclear matter and pion condensation, $\mathrm{Zh}$. Eksp. Teor. Fiz. 66, 443 (1974) [Sov. Phys. JETP 39, 212 (1974)].

[76] A. B. Migdal, Vacuum polarization in strong fields and pion condensation, Sov. Phys. Usp. 20, 879 (1977).

[77] O. V. Bogdanov and P. O. Kazinski, Probability of radiation of twisted photons by axially symmetric bunches of particles, arXiv:1811.12616.

[78] O. V. Bogdanov, P. O. Kazinski, and G. Yu. Lazarenko, Probability of radiation of twisted photons by cold relativistic particle bunches, arXiv:1905.07688.

[79] G. L. Kotkin, V. G. Serbo, and A. Schiller, Processes with large impact parameters at colliding beams, Int. J. Mod. Phys. A 07, 4707 (1992).

[80] D. Seipt, A. Surzhykov, and S. Fritzsche, Structured x-ray beams from twisted electrons by inverse Compton scattering of laser light, Phys. Rev. A 90, 012118 (2014).

[81] A. Angioi, F. Mackenroth, and A. Di Piazza, Nonlinear single Compton scattering of an electron wave packet, Phys. Rev. A 93, 052102 (2016).

[82] D. V. Karlovets, Scattering of wave packets with phases, J. High Energy Phys. 03 (2017) 049.

[83] K. Y. Bliokh et al., Theory and applications of freeelectron vortex states, Phys. Rep. 690, 1 (2017).

[84] S. M. Lloyd, M. Babiker, G. Thirunavukkarasu, and J. Yuan, Electron vortices: Beams with orbital angular momentum, Rev. Mod. Phys. 89, 035004 (2017).

[85] R. J. Glauber, The quantum theory of optical coherence, Phys. Rev. 130, 2529 (1963). 
[86] R. J. Glauber, Coherent and incoherent states of the radiation field, Phys. Rev. 131, 2766 (1963).

[87] J. R. Klauder and E. C. G. Sudarshan, Fundamentals of Quantum Optics (Benjamin, New York, 1968).

[88] M. Tanabashi et al. (Particle Data Group), Review of particle physics, Phys. Rev. D 98, 030001 (2018).

[89] V. Baryshevsky, Channeling, Radiation, and Reactions in Crystals under High Energy (Belarussian State University, Minsk, 1982) [in Russian].

[90] V. Krivitskii and V. Tsytovich, Average radiation-reaction force in quantum electrodynamics, Sov. Phys. Usp. 34, 250 (1991).

[91] Z. Huang, P. Chen, and R. D. Ruth, A semi-classical treatment of channeling radiation reaction, Report No. SLAC-PUB-95-7071, 1995.

[92] P. O. Kazinski and M. A. Shipulya, Asymptotics of physical solutions to the Lorentz-Dirac equation for planar motion in constant electromagnetic fields, Phys. Rev. E 83, 066606 (2011).

[93] T. Schlegel and V. T. Tikhonchuk, Classical radiation effects on relativistic electrons in ultraintense laser fields with circular polarization, New J. Phys. 14, 073034 (2012).

[94] P. O. Kazinski, Radiation of de-excited electrons at large times in a strong electromagnetic plane wave, Ann. Phys. (Amsterdam) 339, 430 (2013).

[95] O. V. Bogdanov and P. O. Kazinski, Properties of electrons scattered by a strong plane electromagnetic wave with a linear polarization: Semiclassical treatment, Pis'ma Zh. Eksp. Teor. Fiz. 101, 224 (2015) [JETP Lett. 101, 206 (2015)].

[96] M. Ruijter, V. Yu. Kharin, and S. G. Rykovanov, Analytical solutions for nonlinear Thomson scattering including radiation reaction, J. Phys. B 51, 225701 (2018).

[97] F. Niel, C. Riconda, F. Amiranoff, M. Lobet, J. Derouillat, F. Pérez, T. Vinci, and M. Grech, From quantum to classical modelling of radiation reaction: A focus on the radiation spectrum, Plasma Phys. Controlled Fusion 60, 094002 (2018).

[98] O. V. Bogdanov, P. O. Kazinski, and G. Yu. Lazarenko, Properties of an ultrarelativistic charged particle radiation in a constant homogeneous crossed electromagnetic field, Ann. Phys. (Amsterdam) 380, 23 (2017).

[99] V. Yanovsky et al., Ultra-high intensity $300 \mathrm{TW}$ laser at $0.1 \mathrm{~Hz}$ repetition rate, Opt. Express 16, 2109 (2008).
[100] O. V. Bogdanov, P. O. Kazinski, and G. Yu. Lazarenko, Probability of radiation of twisted photons in the infrared domain, Ann. Phys. (Amsterdam) 406, 114 (2019).

[101] G. N. Watson, A Treatise on the Theory of Bessel Functions (Cambridge University Press, Cambridge, England, 1944).

[102] O. Matula, A. G. Hayrapetyan, V. G. Serbo, A. Surzhykov, and S. Fritzsche, Radiative capture of twisted electrons by bare ions, New J. Phys. 16, 053024 (2014).

[103] V. Serbo, I. P. Ivanov, S. Fritzsche, D. Seipt, and A. Surzhykov, Scattering of twisted relativistic electrons by atoms, Phys. Rev. A 92, 012705 (2015).

[104] A. A. Peshkov, S. Fritzsche, and A. Surzhykov, Ionization of $\mathrm{H}_{2}^{+}$molecular ions by twisted Bessel light, Phys. Rev. A 92, 043415 (2015).

[105] D. V. Karlovets, G. L. Kotkin, V. G. Serbo, and A. Surzhykov, Scattering of twisted electron wave-packets by atoms in the Born approximation, Phys. Rev. A 95, 032703 (2017).

[106] M. Solyanik-Gorgone, A. Afanasev, C. E. Carlson, C. T. Schmiegelow, and F. Schmidt-Kaler, Excitation of E1forbidden atomic transitions with electric, magnetic, or mixed multipolarity in light fields carrying orbital and spin angular momentum [Invited], J. Opt. Soc. Am. B 36, 565 (2019).

[107] J. Verbeeck, P. Schattschneider, S. Lazar, M. StögerPollach, S. Löffler, A. Steiger-Thirsfeld, and G. Van Tendeloo, Atomic scale electron vortices for nanoresearch, Appl. Phys. Lett. 99, 203109 (2011).

[108] J. C. Kimball and N. Cue, Quantum electrodynamics and channeling in crystals, Phys. Rep. 125, 69 (1985).

[109] J. Augustin, A. Schäfer, and W. Greiner, Quantummechanical treatment of high-energy channeling radiation, Phys. Rev. A 51, 1367 (1995).

[110] H. A. Olsen and Yu. Kunashenko, Dirac states of relativistic electrons channeled in a crystal and high-energy channeling electron-positron pair production by photons, Phys. Rev. A 56, 527 (1997).

[111] I. Bialynicki-Birula and Z. Bialynicka-Birula, Relativistic Electron Wave Packets Carrying Angular Momentum, Phys. Rev. Lett. 118, 114801 (2017).

[112] I. Bialynicki-Birula and Z. Bialynicka-Birula, Twisted localized solutions of the Dirac equation: Hopfion-like states of relativistic electrons, arXiv:1901.01532. 